\title{
Curves and cycles on K3 surfaces
}

\author{
D. Huybrechts
}

With an appendix by C. Voisin

\begin{abstract}
The notion of constant cycle curves on K3 surfaces is introduced. These are curves that do not contribute to the Chow group of the ambient K3 surface. Rational curves are the most prominent examples. We show that constant cycle curves behave in some respects like rational curves. For example, using Hodge theory one finds that in each linear system there are at most finitely many such curves of bounded order. Over finite fields, any curve is expected to be a constant cycle curve, whereas over $\overline{\mathbb{Q}}$ this does not hold. The relation to the Bloch-Beilinson conjectures for K3 surfaces over global fields is discussed.
\end{abstract}

\section{Contents}

1 Introduction $\quad 69$

2 Motivation $\quad 71$

3 Constant cycle curves $\quad \mathbf{7 4}$

4 Constant cycle curves on other surfaces $\quad \mathbf{8 0}$

5 Finiteness of constant cycle curves of fixed order $\quad 81$

6 First examples of constant cycle curves $\quad \mathbf{8 8}$

7 More examples: fixed curves $\quad \mathbf{9 0}$

8 Bitangent correspondence $\quad 92$

9 Finite fields $\quad 97$

10 Further questions and remarks $\quad 99$

$\begin{array}{lr}\text { Appendix by Claire Voisin } & \mathbf{1 0 0}\end{array}$

$\begin{array}{ll}\text { References } & 104\end{array}$

\section{Introduction}

1.1. Due to results of Mumford [Mum68] and Bloch [Blo80], the Chow group of zero-cycles $\mathrm{CH}_{0}(X)=\mathrm{CH}^{2}(X)$ on a complex $\mathrm{K} 3$ surface $X$ is known to be huge (infinite dimensional in some well-defined sense). In particular, there is no curve $C \subset X$ such that the natural push-

Received 11 April 2013, accepted in final form 24 August 2013.

2010 Mathematics Subject Classification 14C25, 14C15

Keywords: K3 surface, Chow group, Bloch-Beilinson conjecture, rational curves.

This journal is (C) Foundation Compositio Mathematica 2014. This article is distributed with Open Access under the terms of the Creative Commons Attribution Non-Commercial License, which permits non-commercial reuse, distribution, and reproduction in any medium, provided that the original work is properly cited. For commercial re-use, please contact the Foundation Compositio Mathematica.

This work was supported by the SFB/TR 45 'Periods, Moduli Spaces and Arithmetic of Algebraic Varieties' of the DFG (German Research Foundation). 


\section{HuYbrechts}

forward map

$$
\operatorname{Pic}(C) \simeq \mathrm{CH}_{0}(C) \rightarrow \mathrm{CH}^{2}(X)
$$

is surjective. This paper studies curves $C \subset X$ for which the image of $\operatorname{Pic}(C) \rightarrow \mathrm{CH}^{2}(X)$ is as small as possible, that is, for which (1.1) induces a trivial map $\operatorname{Pic}^{0}(C) \rightarrow \mathrm{CH}^{2}(X)$.

For a K3 surface over an algebraically closed field $k$, we define an integral curve $C \subset X$ to be a constant cycle curve if the class of its generic point $\eta_{C} \in C$ viewed as a closed point in $X_{k\left(\eta_{C}\right)}=X \times_{k} k\left(\eta_{C}\right)$ satisfies

$$
n \cdot\left[\eta_{C}\right]=n \cdot\left(c_{X}\right)_{k\left(\eta_{C}\right)}
$$

in $\mathrm{CH}^{2}\left(X \times_{k} k\left(\eta_{C}\right)\right)$ for some positive integer $n$. Here, $c_{X} \in \mathrm{CH}^{2}(X)$ is the distinguished class of degree one introduced by Beauville and Voisin in [BV04]. The minimal such $n$ is called the order of the constant cycle curve. If $C \subset X$ is a constant cycle curve, then $\operatorname{Pic}^{0}(C) \rightarrow \mathrm{CH}^{2}(X)$ will be shown to be indeed the zero map. See Sections 3.1 and 3.2 for the definitions and Proposition 3.7 for the relation between the two notions.

Finding sufficient criteria that decide whether a given curve is a constant cycle curve seems as hard as finding criteria that would ensure the opposite. The only positive criterion at the time being is that any rational curve is a constant cycle curve (of order one) and the only effective method to exclude a given curve from being a constant cycle curve uses Hodge theory (cf. Corollary 5.5).

1.2. Some of the results proved in this article are:

- There are at most finitely many constant cycle curves of bounded order in each linear system $|L|$ on a K3 surface $X$ in characteristic zero (cf. Proposition 5.1).

The enumerative problem that suggests itself at this point remains largely open, but see Section 10.2.

To get a better idea of the notion of a constant cycle curve, we give many concrete examples with an emphasis on curves of low order and high genus, see Sections 6-8. Apart from curves of torsion points in families of elliptic curves, the following result turns out to be useful.

- Every fixed curve of a nonsymplectic automorphism is a constant cycle curve (cf. Proposition 7.1).

This immediately leads to the following result.

- There are constant cycle curves of order one that are not rational (cf. Corollary 7.4).

We also manage to construct a constant cycle curve in the generic quartic hypersurface $X \subset \mathbb{P}^{3}$ that is of order at most four and genus 201, see Proposition 8.7.

The notion of constant cycle curves makes sense for arbitrary surfaces. In Section 4, we briefly study surfaces with $p_{g}=0$ satisfying Bloch's conjecture. It can easily be shown that curves on such surfaces are all constant cycle curves of bounded order and that

- on an Enriques surface all curves are constant cycle curves of order at most four (see Proposition 4.2).

In Section 9, we discuss K3 surfaces over finite fields and prove the following result:

- For a Kummer surface $X$ over $\overline{\mathbb{F}}_{p}$, every curve in $X$ is a constant cycle curve (cf. Proposition 9.4). 


\section{CuRves AND CYCLES ON K3 SURFACES}

This is expected to hold for all K3 surfaces over $\overline{\mathbb{F}}_{p}$ and is related to the conjectured finitedimensionality in the sense of Kimura-O'Sullivan and the Bloch-Beilinson conjecture for K3 surfaces over global fields in positive characteristic (see Proposition 9.2). For arbitrary K3 surfaces over $\overline{\mathbb{F}}_{p}$ one can at least prove the following result:

- Let $X$ be a K3 surface over $\overline{\mathbb{F}}_{p}$. Then every closed point $x \in X$ is contained in a constant cycle curve (see Proposition 9.6).

This is expected to hold as well for $X$ over $\overline{\mathbb{Q}}$ and would imply the Bloch-Beilinson conjecture for $X$. For arbitrary K3 surfaces, the existence is expected for points rationally equivalent to points on rational curves. The result should also be compared with [BT05, Thm. 4.2], where it is shown that every point in a Kummer surface over $\overline{\mathbb{F}}_{p}$ associated with the Jacobian of a curve of genus two is contained in a rational curve (and hence in a constant cycle curve of order one).

\section{ACKNOWLEDGEMENTS}

I am grateful to Claire Voisin for many comments, in particular on the torsion problem in the Bloch-Srinivas argument, and for the example in Section 6.3. Thanks to Burt Totaro for a question that triggered the results in Section 9 and for detailed comments on the first version, to Rahul Pandharipande for bringing [GG03] to my attention, and to François Charles and Davesh Maulik for help with arguments related to Section 8. Jimmy Dillies and Alessandra Sarti patiently answered my emails concerning Section 7 and Kieran O'Grady commented on the content of Section 8. Suggestions of the referee have helped to improve the exposition. The intellectual debt to the foundational work of Mark Green, Phillip Griffiths, and Claire Voisin is gratefully acknowledged.

\section{Motivation}

We shall try to motivate the study of constant cycle curves from two perspectives: rational curves and Chow groups. For simplicity we shall restrict ourselves to K3 surfaces over algebraically closed fields $k$ with $\operatorname{char}(k)=0$ and in fact $k=\overline{\mathbb{Q}}$ or $\mathbb{C}$. For technical details, in particular concerning the case $\operatorname{char}(k)>0$, see the later sections.

2.1. Let $(X, H)$ be a polarized complex $\mathrm{K} 3$ surface. The following folklore conjecture has been studied intensively over the last couple of years.

Conjecture 1. The union $\bigcup C \subset X$ of all rational curves $C \subset X$ is dense (in the Zariski or, stronger, classical topology).

The stronger and less studied version would only allow integral rational curves linearly equivalent to some multiple of the given polarization $H$.

The motivation for this conjecture stems from the classical result that for all $m>0$, there exists a rational curve $C$ in the linear system $|m H|$ (Bogomolov, Mumford, Mori-Mukai [MM83]). Here, a curve is rational if the reduction of each of its components has a normalization $\simeq \mathbb{P}^{1}$. For fixed $m>0$ and generic $(X, H)$, that is, for polarized complex K3 surfaces in a Zariski dense open subset of the moduli space of polarized K3 surfaces, $C$ can be chosen integral and even nodal [Che02]. Note that at the same time there are at most finitely many rational curves in any fixed linear system, for example in $|m H|$, as $\mathrm{K} 3$ surfaces in characteristic zero are not 


\section{HuYbrechts}

unirational.

More recently, the conjecture (for the Zariski topology) has been verified for K3 surfaces with $\rho(X) \equiv 1(2)$ by Li and Liedtke [LL12] following an approach by Bogomolov-Hassett-Tschinkel [BHT11]. The same ideas also apply to K3 surfaces that are not defined over $\overline{\mathbb{Q}}$ (see [Huy13] for details). Note that both conditions, ' $\rho(X) \equiv 1(2)$ ' and 'not defined over $\overline{\mathbb{Q}}$ ', are general but not generic, that is, they hold for K3 surfaces in the complement of a countable union of proper algebraic subsets of the moduli space of polarized K3 surfaces. Using work of Bogomolov and Tschinkel [BT00], Chen and Lewis [CL13] settled the conjecture in the classical topology for general $(X, H)$. Not much is known about the stronger form of the conjecture, except for the case $\rho(X)=1$ (when the rational curves have no other choice than being linearly equivalent to multiples of $H)$.

2.2. Assume that $X$ is a $K 3$ surface over $\mathbb{C}$ (or $\overline{\mathbb{Q}}$ ). In [BV04] Beauville and Voisin described a distinguished class $c_{X} \in \mathrm{CH}^{2}(X)$ of degree one, which in particular has the properties that

$$
\mathrm{c}_{2}(X)=24 \cdot c_{X} \text { and } \mathrm{c}_{1}(L)^{2} \in \mathbb{Z} \cdot c_{X}
$$

for all line bundles $L$ on $X$. The set of closed points realizing this class was subsequently studied by McLean in [Mac04], where it is shown that the set

$$
X_{c_{X}}:=\left\{x \in X \mid[x]=c_{X} \in \mathrm{CH}^{2}(X)\right\} \subset X
$$

is dense in the classical topology. Similarly, one can consider the set $X_{\alpha}$ of points realizing any given class $\alpha \in \mathrm{CH}^{2}(X)$ and again, at least for generic $\mathrm{K} 3$ surface, this set is dense if not empty. However, as shall become clear, the set $X_{c_{X}}$ is rather special. For abstract reasons, it is a countable union of Zariski closed subsets, but one expects it to be a countable union of curves, that is, isolated points should not occur. This would be another distinction between $c_{X}$ and any other class $\alpha \in \mathrm{CH}^{2}(X)$ (cf. [Voi12b]). See also Section 3.5 for more on the sets $X_{[x]}$.

The distinguished class $c_{X}$ can also be considered from a more arithmetic point of view, as expressed by the following special case of the much more general set of conjectures due to Bloch and Beilinson. But note that even for K3 surfaces, it has not been verified in a single example.

Conjecture 2 (Bloch-Beilinson). Suppose $X$ is a K3 surface over $\overline{\mathbb{Q}}$ and $x \in X(\overline{\mathbb{Q}})$ is a $\overline{\mathbb{Q}}$ rational point. Then $[x]=c_{X}$.

Note that McLean's proof in fact shows that for every K3 surface $X$ defined over some subfield $k \subset \mathbb{C}$, the set of $\bar{k}$-rational points realizing $c_{X}$ is dense in the classical topology, that is, $X_{c_{X}}(\bar{k}) \subset X(\mathbb{C})$ is dense. In particular, it is known that for $X$ over $\overline{\mathbb{Q}}$ there are many points $x \in X(\overline{\mathbb{Q}})$ realizing $c_{X}$.

The property of $c_{X}$ proved in [BV04] that is the most relevant for our purpose, is the following:

$$
\text { If } x \in C \subset X \text { with } C \text { rational, then }[x]=c_{X} \text {. }
$$

This links rational curves to the study of $\mathrm{CH}^{2}(X)$ and the distinguished class $c_{X} \in \mathrm{CH}^{2}(X)$. In particular, McLean's density result could be seen as (weak) evidence for the density for rational curves as in Conjecture 1. Also, as pointed out by Bogomolov many years ago, it might a priori be possible that any $\overline{\mathbb{Q}}$-rational point lies on a rational curve which in turn would prove Conjecture 2.

The fact that points on rational curves all define the same class in $\mathrm{CH}^{2}(X)$, which eventually relies on the existence of ample rational curves à la Bogomolov-Mumford [MM83], also leads to the concept of constant cycle curves studied in this paper. 


\section{Curves AND CYCles on K3 SuRfaces}

2.3. Let $C \subset X$ be a curve in a complex K3 surface. Then $C$ is called a (pointwise) constant cycle curve if $[x] \in \mathrm{CH}^{2}(X)$ is constant for points $x \in C$ or, equivalently, if the push-forward $\mathrm{Pic}^{0}(C) \rightarrow \mathrm{CH}^{2}(X)$ is the zero map. Voisin shows in [Voi12b], by again using the existence of ample rational curves, that the class realized by a constant cycle curve is always the same, namely $c_{X}$. The most important examples of constant cycle curves are provided by rational curves. But not every (pointwise) constant cycle curve is rational, see Section 6-8 for examples. This triggers the natural question how much weaker the notion of constant cycle curves really is.

As rational curves, constant cycle curves do not come in families (at least not in characteristic zero). Indeed, any family of constant cycle curves would dominate $X$ and so points in an open dense subset would all realize the same class in $\mathrm{CH}^{2}(X)$, contradicting $\mathrm{CH}^{2}(X) \neq \mathbb{Z}$ (cf. the proof of Lemma 3.6, ii)). Hence, for abstract reasons, the set of constant cycle curves in a fixed linear system, for example in $|m H|$, consists of at most countably many points. A finiteness result as for rational curves can be proved after restricting to constant cycle curves of bounded order (see Proposition 5.1). This result is based on normal functions and the recent results of BrosnanPearlstein [BP09] and Saito [Sai08] showing that the zero-set of admissible normal functions is algebraic.

Alternatively to the definition of the order of a constant cycle curve using (1.2), one could define it directly as the order of a certain class $\kappa_{C} \in \mathrm{CH}^{2}\left(X \times k\left(\eta_{C}\right)\right)$ naturally associated to any integral curve $C \subset X$ with its generic point $\eta_{C} \in C$ (see Section 3.2). Note that $\mathrm{CH}^{2}(X)$ is torsion free for $X$ over a separably closed field and so the subtle information needed for the finiteness is contained in the kernel of $\mathrm{CH}^{2}\left(X \times k\left(\eta_{C}\right)\right) \rightarrow \mathrm{CH}^{2}\left(X \times \overline{k\left(\eta_{C}\right)}\right)$.

It is not difficult to show that rational curves are in fact constant cycle curves of order one (see Lemma 6.1). Also, nonrational constant cycle curves can be constructed in many ways, but they usually tend to be of higher order, that is, $\kappa_{C} \neq 0$ in $\mathrm{CH}^{2}\left(X \times k\left(\eta_{C}\right)\right)$. So it is natural to wonder whether constant cycle curves of order one are all rational, but this turns out to be wrong and an explicit counterexample will be described (see Corollary 7.4).

As constant cycle curves of bounded order resemble rational curves in many ways, we state Conjecture 1 for this more flexible class of curves. Note that if in the following the order is not bounded, the result is not difficult to prove, see [Voi12b] or Lemma 6.2.

Conjecture 3. For any $K 3$ surface $X$, there exists an $n>0$ such that the union $\bigcup C \subset X$ of all constant cycle curves $C \subset X$ of order $\leqslant n$ is dense. ${ }^{1}$

Once the finiteness of constant cycle curves of bounded order has been established, it would be interesting to actually count them. Counting rational curves on K3 surfaces is a fascinating subject which recently culminated in the proof of the Yau-Zaslow conjecture in complete generality in [KMPS10]. Unfortunately, it seems much harder to count constant cycle curves, see Section 10.2 .

There is little evidence for an affirmative answer to Bogomolov's question whether any $x \in$ $X(\overline{\mathbb{Q}})$ is contained in a rational curve. Again, one could replace rational curves by constant cycle curves and an affirmative answer to this weaker form would still imply the arithmetic Conjecture 2. The following could be seen as a geometric version.

Conjecture 4 . Let $X$ be a complex $K 3$ surface. Then any point $x \in X$ with $[x]=c_{X}$ is contained in a constant cycle curve.

\footnotetext{
${ }^{1}$ In the appendix Claire Voisin provides a proof of the conjecture for generic complex K3 surfaces. The main idea is to produce constant cycle curves as nontorsion multi-sections of dominating families of elliptic curves, similar to [BT00].
} 


\section{Huybrechts}

As alluded to before, it is much easier to construct constant cycle curves than rational curves. For example, for K3 surfaces over finite fields, every point is in fact contained in a constant cycle curve (see Proposition 9.6). However, a general technique that would allow to settle this problem is not yet available.

\section{Constant cycle curves}

3.1. Let $X$ be a projective $\mathrm{K} 3$ surface over a field $k$. For two reasons, one often has to assume that $k$ is algebraically closed. Firstly, $\mathrm{CH}^{2}(X)$ might have torsion otherwise and, secondly, the good behavior of the Beauville-Voisin class $c_{X}$ depends on the existence of rational curves for which $k$ algebraically closed is needed. In fact, for $k$ not algebraically closed, $c_{X} \in \mathrm{CH}^{2}(X)$ has the desired properties (for example, being realized by points on rational curves) only up to torsion. We will state explicitly when $k=\bar{k}$ is assumed.

Definition 3.1. A curve $C \subset X$ is a pointwise constant cycle curve if all closed points $x \in C$ define the same class $[x] \in \mathrm{CH}^{2}(X)$.

For $k=\bar{k}$ the condition is equivalent to requiring that $[x]=c_{X}$ (the Beauville-Voisin class, see Section 2.2) for all closed points $x \in C$, see [Voi12b, Lem. 2.2]. ${ }^{2}$ Another way of expressing the condition (still assuming $k=\bar{k}$ ) is as follows. A curve $C \subset X$ is a pointwise constant cycle curve if and only if the natural map

$$
f_{C *}: \operatorname{Pic}(\widetilde{C}) \rightarrow \mathrm{CH}^{2}(X)
$$

takes image in $\mathbb{Z} \cdot c_{X}$ or, still equivalently,

$$
f_{C *}: \operatorname{Pic}^{0}(\widetilde{C}) \rightarrow \mathrm{CH}^{2}(X)
$$

is zero. Here, $f_{C}: \widetilde{C} \rightarrow X$ is the composition of the normalization $\widetilde{C} \rightarrow C$ with the inclusion $C \subset X$.

The notion of pointwise constant cycle curves is really interesting only for uncountable fields $k$. For example, it is not preserved under base change when the base field $k$ is too small (cf. Lemma 3.6 and Proposition 3.7). Also, according to Conjecture 2, every curve in a K3 surface over $\overline{\mathbb{Q}}$ should be a pointwise constant cycle curve, which makes it a notion of little interest in this case. However, the same is true for K3 surfaces over $k=\overline{\mathbb{F}}_{p}$ and in this case it is a shadow of the Bloch-Beilinson conjecture for function fields, see Proposition 9.2.

3.2. In order to introduce the finer version of this notion, we define the class $\kappa_{C}$ naturally associated with any integral curve $C \subset X$. To this end, denote (abusively) by $\Delta_{C} \subset X \times C$ the graph of the inclusion and consider the cycle $\Delta_{C}-\left\{x_{0}\right\} \times C$, where $x_{0} \in X$ is an arbitrary point with $\left[x_{0}\right]=c_{X}$. Again, for the existence of such a point and for $c_{X}$ being well-defined, $k$ has to be algebraically closed. Here, $c_{X}$ is the Beauville-Voisin class (cf. Section 2.2). Then let

$$
\kappa_{C} \in \mathrm{CH}^{2}\left(X \times k\left(\eta_{C}\right)\right)
$$

be the class of the restriction $\Delta_{C}-\left\{x_{0}\right\} \times C$ to the generic fibre $X_{k\left(\eta_{C}\right)}=X \times_{k} k\left(\eta_{C}\right)$ of the second projection $X \times C \rightarrow C$. In other words, the generic point $\eta_{C} \in C$ is viewed as a closed

\footnotetext{
${ }^{2}$ We tacitly assume that the standard facts on $c_{X}$ hold true for K3 surfaces over algebraically closed fields of positive characteristic, but all we really need is that points on rational curves all realize the same class. This follows from the existence of ample rational curves, which can be shown by reduction modulo $p$.
} 


\section{Curves AND CYCles on K3 SuRfaces}

point of the K3 surface $X_{k\left(\eta_{C}\right)}$ over the function field $k\left(\eta_{C}\right)$ of $C$ and then corrected by the 'constant' point $\left\{x_{0}\right\} \times \eta_{C}$. This natural class has been considered before in the literature, see for example [Ker06, Ex. 4.2].

Definition 3.2. Assume that $k$ is algebraically closed. An integral curve $C \subset X$ is a constant cycle curve if $\kappa_{C} \in \mathrm{CH}^{2}\left(X \times k\left(\eta_{C}\right)\right)$ is a torsion class.

We call an arbitrary curve $C \subset X$ a constant cycle curve if every integral component of $C$ has this property. Note that this definition makes perfect sense for all surfaces with a distinguished class in $\mathrm{CH}^{2}(X)$, for example for those with $\mathrm{CH}^{2}(X) \simeq \mathbb{Z}$. In fact, constant cycle curves can be defined for arbitrary surfaces by means of Lemma 3.8, but, with the exception of Section 4 , we will restrict ourselves to K3 surfaces.

Remark 3.3. The class $\kappa_{C}$ is in fact the direct image under the push-forward

$$
\mathrm{CH}^{1}\left(C \times k\left(\eta_{C}\right)\right) \rightarrow \mathrm{CH}^{2}\left(X \times k\left(\eta_{C}\right)\right)
$$

of the class $\left[\eta_{C}\right]-\left[x_{0}\right]$, where $x_{0} \in C$ is any point with $\left[x_{0}\right]=c_{X}$ in $\mathrm{CH}^{2}(X)$ (for example a point of intersection with a rational curve). However, the class $\left[\eta_{C}\right]-\left[x_{0}\right] \in \mathrm{CH}^{1}\left(C \times k\left(\eta_{C}\right)\right)$ itself is never torsion except when $C$ is rational, because no nontrivial multiple of $\left[\eta_{C}\right]$ is ever contained in the image of the base change map $\mathrm{CH}^{1}(C) \rightarrow \mathrm{CH}^{1}\left(C \times k\left(\eta_{C}\right)\right)$.

In fact, whether a nonrational curve $C$ is a constant cycle curve depends on the particular embedding $C \hookrightarrow X$. We will see examples of (smooth) curves that can be embedded as constant cycle curves of varying order and even as nonconstant cycle curves in the same K3 surface $X$.

For any field extension $K / k$, the pull-back yields a map

$$
\mathrm{CH}^{2}(X) \rightarrow \mathrm{CH}^{2}\left(X \times_{k} K\right) \text {. }
$$

The image of the Beauville-Voisin class $c_{X} \in \mathrm{CH}^{2}(X)$ shall be denoted $\left(c_{X}\right)_{K}$. It can also be seen, at least for $K$ algebraically closed, as the Beauville-Voisin class of $X_{K}=X \times_{k} K$, that is, $\left(c_{X}\right)_{K}=c_{X_{K}}$. Compare the following result to Lemma 3.8 .

Lemma 3.4. Let $X$ be a K3 surface over an algebraically closed field $k$. For an integral curve $C \subset X$ the following conditions are equivalent:

(i) The curve $C$ is a constant cycle curve.

(ii) There exists a positive integer $n$ such that

$$
n \cdot\left[\eta_{C}\right]=n \cdot\left(c_{X}\right)_{k\left(\eta_{C}\right)}
$$

in $\mathrm{CH}^{2}\left(X \times_{k} k\left(\eta_{C}\right)\right)$, where the generic point $\eta_{C} \in C$ is viewed as a closed point in $X \times_{k}$ $k\left(\eta_{C}\right)$.

(iii) If $\eta_{C} \in C$ is viewed as a point in the geometric generic fibre $X \times_{k} \overline{k\left(\eta_{C}\right)}$, then we have

$$
\left[\eta_{C}\right]=\left(c_{X}\right) \overline{k\left(\eta_{C}\right)}
$$

in $\mathrm{CH}^{2}\left(X \times_{k} \overline{k\left(\eta_{C}\right)}\right)$.

Proof. This is an immediate consequence of the fact that the pull-back (3.4) has torsion kernel and of Roitman's theorem [Roj80] and its generalizations due to Bloch [Blo80] and Milne [Mil82], which show that the group $\mathrm{CH}^{2}\left(X \times_{k} \overline{k\left(\eta_{C}\right)}\right)$ is torsion free. 


\section{Huybrechts}

The Chow group of the generic fibre is best viewed as

$$
\mathrm{CH}^{2}\left(X \times k\left(\eta_{C}\right)\right)=\lim _{\rightarrow} \mathrm{CH}^{2}(X \times U),
$$

where the direct limit is over all nonempty Zariski open subsets $U \subset C$, see [Blo80, Lem. 1.I.20]. Hence, $\kappa_{C}$ is a torsion class if and only if the class $\kappa_{C, U} \in \mathrm{CH}^{2}(X \times U)$ of the restriction of $\left[\Delta_{C}-\left\{x_{0}\right\} \times C\right]$ to $X \times U$ for some nonempty open subset $U \subset C$ is torsion.

In order to obtain finiteness results, one needs to bound the order of the torsion class $\kappa_{C}$ of a constant cycle curve.

Definition 3.5. The order of an integral constant cycle curve $C \subset X$ is the order of the torsion class $\kappa_{C} \in \mathrm{CH}^{2}\left(X \times k\left(\eta_{C}\right)\right)$. The order of an arbitrary constant cycle curve $C$ is the maximal order of its integral components.

Note that by shrinking $U \subset C$, one can always assume that the order of $\kappa_{C}$ and $\kappa_{C, U}$ coincide. By definition, the order is the minimal positive $n$ satisfying (3.5).

3.3. We shall explain the relation between the two notions of constant cycle curves and state some basic properties.

Proposition 3.6. Let $X$ be a K3 surface over an algebraically closed field $k$.

(i) Let $C \subset X$ be a curve and let $K / k$ be an algebraically closed base field extension. Then $C$ is a constant cycle curve of order $n$ if and only if $C_{K} \subset X_{K}$ is a constant cycle curve of order $n$.

(ii) Assume that $X$ is not (Artin) supersingular and that $\operatorname{char}(k) \neq 2 .{ }^{3}$ If $K / k$ is an extension with $K$ also algebraically closed and $D \subset X_{K}$ is a constant cycle curve, then $D$ descends, that is, there exists a constant cycle curve $C \subset X$ with $D=C_{K}$.

(iii) If $X$ is defined over a (finitely generated) field $k_{0}$ with $\bar{k}_{0}=k$ and $C \subset X$ is a constant cycle curve, then the natural $\operatorname{Gal}\left(\bar{k} / k_{0}\right)$-action applied to $C$ yields only constant cycle curves.

Proof. (i) The pull-back

$$
\mathrm{CH}^{2}\left(X \times_{k} k\left(\eta_{C}\right)\right) \rightarrow \mathrm{CH}^{2}\left(X_{K} \times_{K} k\left(\eta_{C_{K}}\right)\right)
$$

induced by the base change $X_{K} \times_{K} k\left(\eta_{C_{K}}\right)=\left(X \times_{k} k\left(\eta_{C}\right)\right) \times_{k} K \rightarrow X \times_{k} k\left(\eta_{C}\right)$ has torsion kernel and maps $\kappa_{C}$ to $\kappa_{C_{K}}$. Hence, $C$ is a constant cycle curve if and only if $C_{K}$ is.

That the order does not change can be shown using arguments of Lecomte in [Lec86], where it is proved that for any variety $Y$ over an algebraically closed field $K_{0}$, the base change

$$
\mathrm{CH}^{*}(Y) \rightarrow \mathrm{CH}^{*}\left(Y_{K}\right)
$$

to a larger algebraically closed field $K / K_{0}$ induces an isomorphism on torsion. (Injectivity is enough for our purpose, for which only $K_{0}$ algebraically closed is needed.) It cannot be applied directly, as in our case $K_{0}=k\left(\eta_{C}\right)$ is not algebraically closed, but we may apply it to $Y=X \times U$

\footnotetext{
${ }^{3}$ This assumption holds whenever $\operatorname{char}(k)=0$; see further on for a reminder on the notion of supersingular K3 surfaces. What is really needed in the proof is $\rho(X) \neq 22$ or $\mathrm{CH}^{2}\left(X_{k^{\prime}}\right) \neq \mathbb{Z}$ for some algebraically closed extension $k^{\prime} / k$. Thanks to Burt Totaro for pointing out how to weaken the original assumption.
} 


\section{Curves AND CyCles ON K3 SuRfaCes}

for open subsets $U \subset C$ to obtain

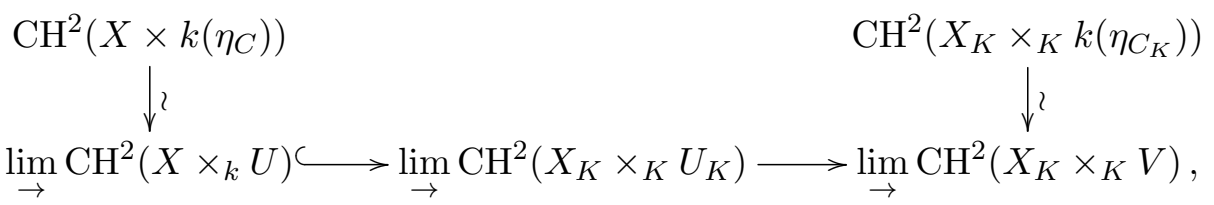

where the last map might a priori not be injective. Suppose that the pull-back $\alpha_{K}$ of $\alpha \in$ $\mathrm{CH}^{2}\left(X \times_{k} U\right)$ yields a trivial class in $\mathrm{CH}^{2}\left(X_{K} \times_{K} V\right)$ for some open set $V \subset U_{K}$, which we can assume to be the complement of finitely many closed points $p_{1}, \ldots, p_{m} \in U_{K}$. (One can further reduce to the case that each of the $p_{i}$ dominates $C$, by shrinking $U$, if necessary). Now use the localization exact sequence (see [Blo80, Ful98, Voi02]):

$$
\mathrm{CH}^{1}\left(X_{K} \times\left\{p_{1}, \ldots, p_{m}\right\}\right) \rightarrow \mathrm{CH}^{2}\left(X_{K} \times_{K} U_{K}\right) \rightarrow \mathrm{CH}^{2}\left(X_{K} \times_{K} V\right) \rightarrow 0
$$

to conclude that $\alpha_{K}$ is supported on $\left\{p_{1}, \ldots, p_{m}\right\}$. Write

$$
\mathrm{CH}^{2}\left(X_{K} \times_{K} U_{K}\right)=\lim \mathrm{CH}^{2}\left(X \times_{k} U \times_{k} W\right)
$$

with the limit over all nonempty open subsets $W \subset \operatorname{Spec}(A)$ for all (finitely generated) $k$-algebras $k \subset A \subset K$ (cf. [Lec86]). For small $W \subset \operatorname{Spec}(A)$, represent $\alpha_{K}$ by $\alpha_{W} \in \mathrm{CH}^{2}\left(X \times_{k} U \times_{k} W\right)$. Its restriction $\alpha_{t}$ to a closed point $t \in W$ gives back $\alpha$. Indeed, since $k$ is algebraically closed, one has $k(t) \simeq k$ and $\alpha \longmapsto \alpha_{W} \longmapsto \alpha_{t}$ is given by the isomorphism $\mathrm{CH}^{2}\left(X \times_{k} U\right) \rightarrow \mathrm{CH}^{2}\left(X \times_{k} U \times_{k} k(t)\right)$. But for small $W \subset \operatorname{Spec}(A)$, the class $\alpha_{t}$ is supported on the intersection of the closure of $\left\{p_{1}, \ldots, p_{m}\right\}$ in $X \times_{k} U \times_{k} W$ with the fibre over $t$, which is a finite set of points $\left\{p_{1 t}, \ldots, p_{m t}\right\}$ in $X \times_{k} U \times_{k} k(t)$. Hence, $\alpha$ restricted to the complement of these points is trivial. This eventually shows that $\alpha$ represents the trivial class in $\mathrm{CH}^{2}\left(X \times k\left(\eta_{C}\right)\right)$.

(ii) If $D \subset X_{K}$ is not defined over $k$, then there exists a one-dimensional family of curves $C_{t} \subset X$ which can be seen as specializations of $D$. In particular, their classes $\kappa_{C_{t}}$ are obtained by specializing $\kappa_{D}$ and, therefore, are also torsion. Thus, one would obtain a dominant family of pointwise constant cycle curves $C_{t} \subset X$. For every larger algebraically closed $k^{\prime} / k$ base changing, the family $\left\{C_{t}\right\}$ defines a dominating family of constant cycle curves for $X_{k^{\prime}}$. This would imply that $\mathrm{CH}^{2}\left(X_{k^{\prime}}\right) \simeq \mathbb{Z}$, which is excluded for nonsupersingular $\mathrm{K} 3$ surfaces, such as $\mathrm{CH}^{2}(X \times$ $\left.\overline{k\left(\eta_{X}\right)}\right) \neq \mathbb{Z}$. Recall that a K3 surface $X$ over a field $k$ of $\operatorname{char}(k)>0$ is called Artin supersingular if its height is infinite and Shioda supersingular if $\rho\left(X_{\bar{k}}\right)=22$. It has been known for a long time that Shioda supersingular implies Artin supersingular and the proof of the converse has recently be completed in [Cha12, Mau12, Mad13]. (It has also been known that unirational K3 surfaces are Shioda (and hence Artin) supersingular and the converse has been established in [Lie13].) Thus, a K3 surface $X$ over $k$ with $\operatorname{char}(k) \neq 2$ is supersingular if and only if $\rho(X) \neq 22$, that is, $H_{e t}^{2}\left(X, \mathbb{Q}_{\ell}(1)\right) \neq \mathrm{NS}(X) \otimes \mathbb{Q}_{\ell}$. Hence Bloch's result [Blo80, Thm. 6, Appendix to Sec. 1] applies.

(iii) The last assertion is obvious, as the notion of constant cycle curves is scheme-theoretic.

For the reader's convenience and later use, we recall the following fact, which is a special case of a result due to Voisin (cf. [Voi02, Ch. 22]) improving upon a result of Bloch and Srinivas [BS83].

Proposition 3.7. Assume that $k$ is algebraically closed. Then a constant cycle curve $C \subset X$ is also a pointwise constant cycle curve. If $k$ is uncountable, the converse holds true as well. 


\section{Huybrechts}

Proof. Clearly, we may assume that $C$ is integral and for simplicity we also assume that $C$ is smooth (otherwise we pass to its normalization and replace $C \hookrightarrow X$ by the generically finite map $\widetilde{C} \longrightarrow X)$. The first assertion is easy. If $\kappa_{C}$ is torsion, say $n \cdot \kappa_{C}=0$, then there exists an open subset $U:=C \backslash\left\{p_{1}, \ldots, p_{m}\right\} \subset C$ such that $0=n \cdot\left[\left.\left(\Delta_{C}-\left\{x_{0}\right\} \times C\right)\right|_{X \times U}\right] \in \mathrm{CH}^{2}(X \times U)$ (use (3.6)). By the localization exact sequence

$$
\mathrm{CH}^{1}\left(X \times\left\{p_{1}, \ldots, p_{m}\right\}\right) \rightarrow \mathrm{CH}^{2}(X \times C) \rightarrow \mathrm{CH}^{2}(X \times U) \rightarrow 0
$$

we can assume that $n \cdot\left(\Delta_{C}-\left\{x_{0}\right\} \times C\right)$ is rationally equivalent to a cycle $Z$ on $X \times C$ with support in $X \times\left\{p_{1}, \ldots, p_{m}\right\}$. As any zero-cycle on $C$ is linearly equivalent to one disjoint to the finite set of points $\left\{p_{1}, \ldots, p_{m}\right\}$, the induced map $[Z]_{*}: \mathrm{CH}_{0}(C) \rightarrow \mathrm{CH}^{2}(X)$ is trivial. Thus, $n \cdot\left[\Delta_{C}-\left\{x_{0}\right\} \times C\right]_{*}: \mathrm{CH}_{0}(C) \rightarrow \mathrm{CH}^{2}(X)$ is trivial and, since $\mathrm{CH}^{2}(X)$ is torsion free, we also have $\left[\Delta_{C}-\left\{x_{0}\right\} \times C\right]_{*}=0$. The latter is equivalent to saying that $[x] \equiv\left[x_{0}\right]=c_{X}$ for all closed points $x \in C$.

For the converse, use [Voi02, Cor. 22.20], which is stated for $k=\mathbb{C}$ but in fact holds for any uncountable field $k$. Then $n \cdot\left[\Delta_{C}-\left\{x_{0}\right\} \times C\right]=[Z]$ for some $n>0$ with $Z$ supported on a closed set of the form $X \times\left\{p_{1}, \ldots, p_{m}\right\}$. But then $\left.Z\right|_{X_{k\left(\eta_{C}\right)}}=0$ and hence $\kappa_{C} \in \mathrm{CH}^{2}\left(X \times k\left(\eta_{C}\right)\right)$ is torsion.

3.4. One could avoid mentioning the distinguished class $c_{X} \in \mathrm{CH}^{2}(X)$ in the definition of a constant cycle curve altogether by proving the next result, analogous to Lemma 3.4.

Lemma 3.8. Let $X$ be a K3 surface over an algebraically closed field $k$. For an integral curve $C \subset X$ the following conditions are equivalent:

(i) The curve $C$ is a constant cycle curve.

(ii) There exists a positive integer $n$ such that

$$
n \cdot\left[\eta_{C}\right] \in \operatorname{Im}\left(\mathrm{CH}^{2}(X) \rightarrow \mathrm{CH}^{2}\left(X \times_{k} k\left(\eta_{C}\right)\right)\right),
$$

where the generic point $\eta_{C} \in C$ is viewed as a closed point in $X \times_{k} k\left(\eta_{C}\right)$.

(iii) If $\eta_{C} \in C$ is viewed as a point in the geometric generic fibre $X \times_{k} \overline{k\left(\eta_{C}\right)}$, then

$$
\left[\eta_{C}\right] \in \operatorname{Im}\left(\mathrm{CH}^{2}(X) \rightarrow \mathrm{CH}^{2}\left(X \times_{k} \overline{k\left(\eta_{C}\right)}\right)\right) .
$$

Proof. Clearly, condition (i) implies conditions (ii) and (iii). Conditions (ii) and (iii) are equivalent because $\mathrm{CH}^{2}\left(X \times_{k} k\left(\eta_{C}\right)\right) \rightarrow \mathrm{CH}^{2}\left(X \times_{k} \overline{k\left(\eta_{C}\right)}\right)$ has torsion kernel and torsion free target.

It remains to show that condition (ii) implies condition (i). Now, for a closed point $x \in C$, the composition of the specialization map $s_{x}: \mathrm{CH}^{2}\left(X \times k\left(\eta_{C}\right)\right) \rightarrow \mathrm{CH}^{2}(X)$ (see [Ful98, Ch. 20.3]) with the pull-back (3.9) yields the identity on $\mathrm{CH}^{2}(X)$. If now $n \cdot\left[\eta_{C}\right]$ is in the image of (3.9), say $n \cdot\left[\eta_{C}\right]=\alpha_{k\left(\eta_{C}\right)}$, then $n \cdot[x]=\alpha$, as $\eta_{C}$ clearly specializes to $[x]$. Thus, $C$ is a pointwise constant cycle curve, which for an uncountable field is enough to conclude (use Proposition 3.7).

If $k$ is only countable, use base change to an uncountable algebraically closed extension $K / k$ (for example a universal domain). Clearly, then $n \cdot\left[\eta_{C_{K}}\right]=n \cdot\left[\eta_{C}\right]_{K}$ is contained in the image of $\mathrm{CH}^{2}(X) \longrightarrow \mathrm{CH}^{2}\left(X_{K} \times_{K} k\left(\eta_{C_{K}}\right)\right)$. Hence, $C_{K}$ is a constant cycle curve and, by Lemma 3.6 (i), $C$ also is.

3.5. Let $K / k$ be an extension of algebraically closed fields. Let $X$ be a K3 surface over $k$ and denote by $X_{K}$ the K3 surface over $K$ obtained by base change. The base change morphism $\xi: X_{K} \longrightarrow X$ induces the pull-back map $\xi^{*}: \mathrm{CH}^{2}(X) \hookrightarrow \mathrm{CH}^{2}\left(X_{K}\right)$, that is, $[x]_{K}=\xi^{*}[x]$ for all 


\section{Curves And CYCles on K3 SuRfaCes}

closed points $x \in X$. It is injective, but not surjective as soon as $\operatorname{trdeg}_{k}(K) \geqslant 2$ and $\rho(X) \neq 22$ (see [Blo80]) or $\operatorname{trdeg}_{k}(K) \geqslant 1$ and $\operatorname{char}(k)=0$ (see [GGP04]).

Compare the following to [Gor12, Prop. 5].

Corollary 3.9. Let $x \in X_{K}$ be a closed point with

$$
[x] \in \operatorname{Im}\left(\mathrm{CH}^{2}(X) \rightarrow \mathrm{CH}^{2}\left(X_{K}\right)\right),
$$

for example $[x]=c_{X_{K}}=\left(c_{X}\right)_{K}$. Then one of the following is true:

(i) The image $\xi(x) \in X$ is a closed point, that is, $x$ is defined over $k$.

(ii) The closure $C:=\overline{\{\xi(x)\}} \subset X$ of $\xi(x) \in X$ is a constant cycle curve.

(iii) The image $\xi(x) \in X$ is the generic point of $X$ and $\mathrm{CH}^{2}(X) \simeq \mathbb{Z}$.

Proof. Suppose that $x$ is not defined over $k$. Then its image in $X$ is either the generic point of $X$ or that of a curve $C \subset X$. In the second case, consider the natural inclusion $k\left(\eta_{C}\right) \hookrightarrow k(x) \simeq K$ for the generic point $\eta_{C} \in C$. The induced map $\mathrm{CH}^{2}\left(X \times k\left(\eta_{C}\right)\right) \rightarrow \mathrm{CH}^{2}\left(X_{K}\right)$ sends $\left[\eta_{C}\right]$ to $[x]$. Since the kernel is torsion, one can conclude by Lemma 3.8. Similarly, if $x$ is mapped to $\eta_{X} \in X$, then, up to torsion, $\left[\eta_{X}\right]$ is contained in the image of $\mathrm{CH}^{2}(X) \rightarrow \mathrm{CH}^{2}\left(X \times_{k} k\left(\eta_{X}\right)\right)$. And then, by specialization, we in fact have $[y] \equiv$ const for all points $y \in X$.

So, in principle, one could try to produce constant cycle curves by finding points $x \in X_{K}$ with $[x]=c_{X_{K}}$ not defined over $k$. Then either $\mathrm{CH}^{2}(X) \simeq \mathbb{Z}$ or the closure of $x$ in $X$ is a constant cycle curve. Although finding points $x$ not defined over $k$ is in principle possible, deciding whether $[x]=c_{X_{K}}$ also holds is difficult to verify without knowing beforehand that $x$ is contained in a constant cycle curve $C \subset X_{K}$ which for $\mathrm{CH}^{2}(X) \nsucceq \nsucceq \mathbb{Z}$ would automatically descend to $k$, cf. Lemma 3.6. Also note that by Lemma 3.6 (i), one might expect that the order of the constant cycle curve $C:=\overline{\{\xi(x)\}} \subset X$ in situation (ii) is an invariant of the point $x \in X_{K}$, but how to read it off directly from $x$ is unclear.

Corollary 3.10. Let $x \in X_{K}$ be a closed point not defined over $k$. Then

(i) either $[x]=\left(c_{X}\right)_{K} \in \mathrm{CH}^{2}\left(X_{K}\right)$ and there exists a constant cycle curve $x \in C \subset X_{K}$

(ii) or the class $[x]$ is not contained in the image of $\mathrm{CH}^{2}(X) \rightarrow \mathrm{CH}^{2}\left(X_{K}\right)$.

Proof. Indeed, take the curve $\overline{\{\xi(x)\}} \subset X$ (or any curve contained in it) and base change it to a curve in $X_{K}$.

Rephrasing this result yields another proof of a weak form of the main result of [GGP04] for K3 surfaces and of the original result by Bloch [Blo80]. Contrary to the proof in [GGP04], the arguments here do not involve Hodge theory and, therefore, work in positive characteristic. Roughly, the result says that if $\mathrm{CH}^{2}(X)_{0} \neq 0$, then $\mathrm{CH}^{2}(X)$ grows under any base field extension to a bigger algebraically closed field.

Corollary 3.11. Let $K / k$ be an extension of algebraically closed fields with $\operatorname{trdeg}_{k}(K)>0$. If $X$ is a $K 3$ surface over $k$ with $\mathrm{CH}^{2}(X) \not \mathbb{Z}$, then

$$
\mathrm{CH}^{2}(X) \rightarrow \mathrm{CH}^{2}\left(X_{K}\right)
$$

is not surjective.

Remark 3.12. The following is rather speculative and probably well-know to experts: One may wonder, whether the above opens a way to prove the Bloch-Beilinson conjecture for K3 surfaces 


\section{Huybrechts}

(see Conjecture 2). Suppose that $X$ is a K3 surface over $k=\overline{\mathbb{Q}}$. If for any $x_{0} \in X(k)$, there exist a field extension $K / k$ and a point $x \in X(K) \backslash X(k)$ with $[x]=\left[x_{0}\right]_{K}$, then $\mathrm{CH}^{2}(X) \simeq \mathbb{Z}$. Indeed, by Corollary 3.10 one would have $[x]=\left(c_{X}\right)_{K}$, as $[x]$ is by assumption contained in the image of $\mathrm{CH}^{2}(X) \rightarrow \mathrm{CH}^{2}\left(X_{K}\right)$, and hence $\left[x_{0}\right]=c_{X}$. Unfortunately, I do not know of any method that could possibly construct such a point $[x]$. Note that it has to be important that the original surface is defined over a number field, that is, $k=\overline{\mathbb{Q}}$, as we do not expect $\mathrm{CH}^{2}(X) \simeq \mathbb{Z}$ for $X$ over bigger fields. (A concrete counterexample has been given by Schoen, see [Jan07].)

Similarly to (2.2) one can define for any point $x_{0} \in X(k)$ the set

$$
X_{\left[x_{0}\right]}(k):=\left\{x \in X(k) \mid[x]=\left[x_{0}\right] \in \mathrm{CH}^{2}(X)\right\},
$$

which due to Maclean's result [Mac04] is dense (at least for generic complex $X$ ). If $C \subset X$ is a constant cycle curve, then $C(k) \subset X_{c_{X}}(k)$. Now consider a nontrivial extension $K / k$ with $K$ algebraically closed. Then $C_{K} \subset X_{K}$ remains a constant cycle curve. Clearly, not all points in $C_{K}$ will be defined over $k$ and, therefore, the natural inclusion

$$
X_{c_{X}}(k) \subset X_{c_{X}}(K)
$$

is strict. For any other class $\left[x_{0}\right] \neq c_{X}$, the set of points realizing it does not grow under base field extension, as shown by the next result which is again just a reformulation of Corollary 3.10.

Corollary 3.13. Let $K / k$ be any extension of algebraically closed fields. If $X$ is a K3 surface over $k$ and $x_{0} \in X$ is a closed point with $\left[x_{0}\right] \neq c_{X}$, then

$$
X_{\left[x_{0}\right]}(k)=X_{\left[x_{0}\right]}(K),
$$

that is, all points in $X_{K}$ rationally equivalent to $x_{0}$ are defined over $k$.

\section{Constant cycle curves on other surfaces}

The notion of constant cycle curves makes sense for other types of surfaces, see Section 3.2. We shall briefly discuss the case of surfaces satisfying Bloch's conjecture. Recall that Bloch's conjecture for surfaces $X$ with $p_{g}(X)=0$ predicts that the kernel of the Albanese map

$$
\mathrm{CH}^{2}(X)_{0} \rightarrow \operatorname{Alb}(X)
$$

is trivial. It has been verified in [BKL76] for all surfaces of Kodaira dimension $\leqslant 1$ and for many surfaces of general type, in particular those dominated by products of curves. In [Kim05, Cor. 7.7] it has been shown that the finite-dimensionality of the Chow motive $\mathfrak{h}(X)$ (in the sense of Kimura-O'Sullivan) implies Bloch's conjecture. For simplicity we will restrict to the case $q(X)=0$, otherwise one would have to restrict ourselves to curves in the fibres of the Albanese map.

Proposition 4.1. Let $X$ be a smooth projective surface with $p_{g}(X)=q(X)=0$ over an algebraically closed field $k$ of characteristic zero. Then $X$ satisfies Bloch's conjecture, that is, $\mathrm{CH}^{2}(X)_{0}=0$, if and only if every curve in $X$ is a constant cycle curve.

Proof. Suppose $X$ satisfies Bloch's conjecture. If $k$ is uncountable, for example $k=\mathbb{C}$, then the assertion follows from the arguments used to prove Proposition 3.7. Indeed, assuming Bloch's conjecture, every curve is a pointwise constant cycle curve. For arbitrary field $k$ one uses [GP03, Thm. 7] (or directly the techniques of [BS83]), which states that Bloch's conjecture is equivalent to the finite-dimensionality of $\mathfrak{h}(X)$. By [KMP07], the Chow motive $\mathfrak{h}(X)$ is finite-dimensional 


\section{Curves AND CYCles on K3 SuRfaces}

if and only if its transcendental motive $\mathfrak{t}^{2}(X)$ is finite-dimensional. But for $p_{g}(X)=0$, the latter is equivalent to $\mathfrak{t}^{2}(X)=0$ and by [KMP07, Cor. 7.4.9], this implies $\mathrm{CH}^{2}\left(X \times k\left(\eta_{X}\right)\right)_{0} \otimes \mathbb{Q}=0$ (see also the discussion in [And05, Ch. 4.1]). To conclude, use that the generic point of any curve $C \subset X$ is a specialization of $\eta_{X}$. Conversely, if every curve $C \subset X$ is a constant cycle curve, then clearly $\mathrm{CH}^{2}(X) \simeq \mathbb{Z}$.

However, determining the order of constant cycle curves in these surfaces is a different matter. Mainly, because nothing seems to be known about the integral version of the transcendental motive $\mathfrak{t}^{2}(X)$ and the torsion in $\mathrm{CH}^{2}\left(X \times k\left(\eta_{X}\right)\right)$ is difficult to control.

The following cases are instructive for our purpose.

Proposition 4.2. Let $X$ be a surface over an algebraically closed field $k$ with $p_{g}(X)=q(X)=0$ satisfying Bloch's conjecture $\mathrm{CH}^{2}\left(X_{K}\right)_{0}=0$ for all algebraically closed extensions $K / k$.

(i) There exists an integer $n$ such that all curves on $X$ are constant cycle curves of order $\leqslant n .{ }^{4}$

(ii) If $X$ is rational, then every curve $C \subset X$ is a constant cycle curve of order one.

(iii) If $X$ is an Enriques surface, then all curves are constant cycle curves of order $n \mid 4$.

Proof. (i) For an integral curve $C \subset X$, consider the specialization map

$$
\mathrm{CH}^{2}\left(X \times k\left(\eta_{X}\right)\right) \rightarrow \mathrm{CH}^{2}\left(X \times k\left(\eta_{C}\right)\right)
$$

which sends $\left[\eta_{X}\right]$ to $\left[\eta_{C}\right]$. Thus, it suffices to show that there exists an $n$ with $n \cdot\left[\eta_{X}\right]=$ $n \cdot\left[x_{0} \times k\left(\eta_{X}\right)\right]$ for some $x_{0} \in X$ or, equivalently, that $\left[\eta_{X}\right]-\left[x_{0} \times k\left(\eta_{X}\right)\right]$ is contained in the kernel of $\mathrm{CH}^{2}\left(X \times k\left(\eta_{X}\right)\right) \rightarrow \mathrm{CH}^{2}\left(X \times \overline{k\left(\eta_{X}\right)}\right)$. But by assumption, $\mathrm{CH}^{2}\left(X \times \overline{k\left(\eta_{X}\right)}\right) \simeq \mathbb{Z}$ and $\left[\eta_{X}\right]-\left[x_{0} \times k\left(\eta_{X}\right)\right]$ is of degree zero.

(ii) As rational curves are constant cycle curves of order one (see Lemma 6.1 for the argument, which is a direct consequence of the definition), we may also replace $X$ by a minimal model.

So, if $X$ is rational, we can in fact assume that $X \simeq \mathbb{P}^{2}$. Now use that $\left[\Delta_{\mathbb{P}^{2}}\right] \in \mathrm{CH}^{2}\left(\mathbb{P}^{2} \times \mathbb{P}^{2}\right)$ can be written as $\left[\Delta_{\mathbb{P}^{2}}\right]=h_{1}^{2} \times 1+h_{1} \times h_{2}+1 \times h_{2}^{2}$, where the $h_{i}, i=1,2$, denote the hyperplane sections on the two factors. Hence, for every integral curve $C \subset \mathbb{P}^{2}$, one finds $\left[\Delta_{C}\right]=\left.\left[\Delta_{\mathbb{P}^{2}}\right]\right|_{\mathbb{P}^{2} \times C}=$ $h_{1}^{2} \times[C]+h_{1} \times\left. h_{2}\right|_{C}$ and, therefore, $\kappa_{C}=0$.

(iii) Every Enriques surface admits an elliptic fibration $X \rightarrow \mathbb{P}^{1}$ with a 2-section $C_{0} \subset X$. Therefore the base change to $X \times k\left(\eta_{C}\right)$ for any curve $C \subset X$ comes with a natural 2-section, too. Now copy the arguments in [BKL76] to show that every class in $\mathrm{CH}^{2}\left(X^{\prime}\right)_{0}$ for an Enriques surface $X^{\prime}$ over an arbitrary field $k^{\prime}$ is annihilated by $n^{2}$, when $n$ is the degree of a multi-section. In [BKL76] this was combined with the fact that $\mathrm{CH}^{2}\left(X^{\prime}\right)_{0}$ is torsion free for $k^{\prime}$ algebraically closed to deduce that $\mathrm{CH}^{2}\left(X^{\prime}\right)_{0}=0$. Here, we apply it to $X^{\prime}=X \times k\left(\eta_{C}\right)$ and $k^{\prime}=k\left(\eta_{C}\right)$ to show the assertion.

\section{Finiteness of constant cycle curves of fixed order}

The aim of the section is to prove the following result.

Proposition 5.1. Let $X$ be a projective K3 surface over an algebraically closed field of characteristic zero. Then there are at most finitely many constant cycle curves $C$ of fixed order $n$ in any linear system $|L|$.

\footnotetext{
${ }^{4}$ Thanks to Claire Voisin for suggesting this.
} 


\section{Huybrechts}

It is clearly enough to prove the theorem for complex K3 surfaces. The techniques, involving Hodge theory and normal functions, do not apply to K3 surfaces over fields of positive characteristic. In fact, for unirational $\mathrm{K} 3$ surfaces over $\overline{\mathbb{F}}_{p}$, which come with infinitely many rational and hence constant cycle curves of order one in a certain linear system, the result is clearly false. Whether this is the only exception is not clear.

5.1. For the Hodge-theoretic considerations below, we first need to introduce a compactification of $\kappa_{C}$ to a class in $\mathrm{CH}^{2}(X \times C)$ that is different from the naive one used for example in the proof of Proposition 3.7.

Let $X$ be a K3 surface with a fixed point $x_{0} \in X$ such that $\left[x_{0}\right]=c_{X}$. Consider an integral curve $C \subset X$ and its normalization $\widetilde{C} \rightarrow C$. As before, the composition shall be denoted by $f_{C}: \widetilde{C} \rightarrow C \subset X$. Given $C$ and cycle $\sum n_{i} \cdot y_{i}$ of degree one on $\widetilde{C}$, one defines

$$
Z_{C}:=\Delta_{f_{C}}-C \times\left(\sum n_{i} \cdot y_{i}\right)-\left\{x_{0}\right\} \times \widetilde{C},
$$

which is an integral cycle on the smooth threefold $X \times \widetilde{C}$. Here, $\Delta_{f_{C}}$ denotes the graph of $f_{C}$, which can also be seen as the pull-back of the diagonal $\Delta_{X} \subset X \times X$ under (id, $\left.f_{C}\right): X \times \widetilde{C} \rightarrow X \times X$. Note that, although not reflected by the notation, $Z_{C}$ and the associated class $\left[Z_{C}\right] \in \mathrm{CH}^{2}(X \times \widetilde{C})$ do depend on the cycle $\sum n_{i} \cdot y_{i}$ and the associated line bundle $L_{0}:=\mathcal{O}\left(\sum n_{i} \cdot y_{i}\right)$ on $\widetilde{C}$, respectively. (In Lemma 5.4 below, the transcendental Abel-Jacobi class associated with $Z_{C}$ will be shown to be independent of $\sum n_{i} \cdot y_{i}$.) Geometrically, it would seem natural to choose $L_{0}$ to be of the form $\mathcal{O}\left(y_{0}\right)$ for some point $y_{0} \in \widetilde{C}$. However, for arguments involving families of curves $C$, allowing line bundles has technical advantages, cf. Section 5.3. In fact, we will later choose $L_{0}$ such that $L_{0}^{2 g-2} \simeq f_{C}^{*} L$, for $C \in|L|$ with $L . L=2 g-2$.

It is straightforward to check that $Z_{C}$ is homologously trivial, for example for a complex K3 surface, the induced map

$$
\left[Z_{C}\right]_{*}: H^{*}(\widetilde{C}, \mathbb{Z}) \rightarrow H^{*}(X, \mathbb{Z})
$$

is zero. This allows one to define its Abel-Jacobi class, see Section 5.2.

For an integral curve $C$, the generic points $\eta_{\widetilde{C}} \in \widetilde{C}$ and $\eta_{C} \in C$ can be identified. We have the following result.

Lemma 5.2. For an integral curve $C \subset X$, restriction to the generic fibre maps $\left[Z_{C}\right]$ to $\kappa_{C}$, that is,

$$
\mathrm{CH}^{2}(X \times \widetilde{C}) \longrightarrow \mathrm{CH}^{2}\left(X \times k\left(\eta_{C}\right)\right), \quad\left[Z_{C}\right] \longmapsto \kappa_{C} .
$$

Clearly, if $\left[Z_{C}\right]$ is torsion, then so is $\kappa_{C}$ and hence $C$ is a constant cycle curve, but the converse is not true. If in addition $L_{0}^{2 g-2} \simeq f_{C}^{*} L$ is required, then at least the question whether the cycle $Z_{C}$ is torsion does no longer depend on the particular $L_{0}$, for another choice of $L_{0}$ differs by torsion in $\operatorname{Pic}^{0}(\widetilde{C})$.

Remark 5.3. We briefly discuss various other possible choices for $Z_{C}$. We restrict ourselves to the case of a complex K3 surface $X$.

(i) On $X \times X$ one often considers

$$
Z_{X}:=\Delta_{X}-\sum n_{i} \cdot\left(C_{i} \times D_{i}\right)-\left\{x_{0}\right\} \times X-X \times\left\{x_{0}\right\}
$$

the transcendental part of the diagonal. The curves $C_{i}, D_{i} \subset X$ and the $n_{i} \in \mathbb{Q}$ are chosen such that $[Z]_{*}$ is trivial on $H^{0}(X, \mathbb{Q}) \oplus H^{4}(X, \mathbb{Q}) \oplus \mathrm{NS}(X) \otimes \mathbb{Q} \subset H^{*}(X, \mathbb{Q})$, that is, such that 


\section{CuRves AND CYCLES ON K3 SURFACES}

$\left[Z_{X}\right] \in T(X) \otimes T(X) \otimes \mathbb{Q} \subset H^{4}(X \times X, \mathbb{Q})$, where $T(X) \subset H^{2}(X, \mathbb{Z})$ is the transcendental lattice. For $\rho(X)=1$, this can be rewritten as

$$
Z_{X}=\Delta_{X}-(1 /(2 g-2)) \cdot(C \times C)-\left\{x_{0}\right\} \times X-X \times\left\{x_{0}\right\},
$$

with $C . C=2 g-2$, which is defined in general and satisfies $(2 g-2) \cdot\left[Z_{X}\right] \in[C]^{\perp} \otimes[C]^{\perp} \subset$ $H^{2}(X, \mathbb{Z}) \times H^{2}(X, \mathbb{Z})$.

The pullback to $X \times \widetilde{C}$ would be another natural choice for $Z_{C}$ with the property that its restriction to $X \times k\left(\eta_{C}\right)$ represents $\kappa_{C}$. The obvious advantage to being defined universally on $X \times X$ has the price, due to the coefficient $1 /(2 g-2)$, that $Z_{C}$ is only rationally defined, that is, $\left[\left(\mathrm{id}, f_{C}\right)^{*} Z_{X}\right]$ is well defined only in $\mathrm{CH}^{2}(X \times \widetilde{C}) \otimes \mathbb{Q}$, which makes it more difficult to work with its Abel-Jacobi class.

Similarly, the class defined by the cycle

$$
Z_{C}^{\prime}:=\Delta_{f_{C}}-(1 /(2 g(\widetilde{C})-2)) \cdot(C \times D)-\left\{x_{0}\right\} \times C
$$

with $D \in\left|\omega_{C}\right|$ would be independent of any additional choice of $L_{0} \in \operatorname{Pic}^{1}(\widetilde{C})$, but again it is only rational.

(ii) In [GG03] Green and Griffiths study a cycle introduced by Faber and Pandharipande. For a divisor $D$ of degree $d$ on a smooth curve $C$, they define

$$
z_{D}:=D \times D-d \cdot D_{\Delta} .
$$

Assume now that $C$ is contained in a K3 surface $X$ and suppose for simplicity that $C$ generates $\operatorname{NS}(X)$. Then for $D \in\left|\omega_{C}\right|$,

$$
\left.z_{D} \sim(2-2 g(C)) \cdot Z_{X}\right|_{C \times C}
$$

under $C \times C \subset X \times X$, where in this case $Z_{X}$ is as in (5.2). Similarly, $z_{D}$ is rationally equivalent to the restriction of $(2-2 g(C)) \cdot Z_{C}^{\prime}$ under $C \times C \subset X \times C$.

As pointed out in [GG03], the class $\left[z_{D}\right] \in \mathrm{CH}^{2}(C \times C) \otimes \mathbb{Q}$ for $D \in\left|\omega_{C}\right|$ is (by Bloch-Beilinson) conjectured to be trivial for curves $C$ defined over $\overline{\mathbb{Q}}$, but it is also shown that it is not trivial for general $C$ of genus $g(C) \geqslant 4$ (see also the shorter proof in [Yin13]). For $4 \leqslant g(C)<10$ this can be used to show that for general $C \subset X$ (with varying $X$ ) the class $\left[Z_{C}^{\prime}\right] \in \mathrm{CH}^{2}(X \times C) \otimes \mathbb{Q}$ is nontrivial.

5.2. Consider the Abel-Jacobi map for the smooth threefold $X \times \widetilde{C}$ :

$$
\text { AJ }: \mathrm{CH}^{2}(X \times \widetilde{C})_{\mathrm{hom}} \rightarrow J^{3}(X \times \widetilde{C}), \quad \gamma \mapsto \int_{\Gamma} .
$$

Here, $\mathrm{CH}^{2}(X \times \widetilde{C})_{\text {hom }}$ denotes the homologically trivial part and for any $\gamma$, we let $\Gamma$ be a real three-dimensional cycle with $\partial \Gamma=\gamma$. The intermediate Jacobian

$$
J^{3}(X \times \widetilde{C}):=\frac{H^{3}(X \times \widetilde{C}, \mathbb{C})}{F^{2} H^{3}(X \times \widetilde{C})+H^{3}(X \times \widetilde{C}, \mathbb{Z})} \simeq \frac{F^{2} H^{3}(X \times \widetilde{C})^{*}}{H^{3}(X \times \widetilde{C}, \mathbb{Z})}
$$

is a complex torus of dimension $22 \cdot g(\widetilde{C})$.

We shall also need the 'transcendental part' of the intermediate Jacobian, which we define as (see also [Gre98, Voi99]):

$$
J^{3}(X \times \widetilde{C})_{\operatorname{tr}}:=\frac{F^{2}\left(T(X) \otimes H^{1}(\widetilde{C}, \mathbb{C})\right)^{*}}{T(X) \otimes H^{1}(\widetilde{C}, \mathbb{Z})} .
$$




\section{Huybrechts}

Here, $T(X):=\mathrm{NS}(X)^{\perp} \subset H^{2}(X, \mathbb{Z})$ is the transcendental lattice, which equals the orthogonal complement $[C]^{\perp} \subset H^{2}(X, \mathbb{Z})$ if $\rho(X)=1$. We shall denote the composition of the projection $J^{3}(X \times \widetilde{C}) \rightarrow J^{3}(X \times \widetilde{C})_{\text {tr }}$ with the Abel-Jacobi map by

$$
\mathrm{AJ}_{\mathrm{tr}}: \mathrm{CH}^{2}(X \times \widetilde{C})_{\mathrm{hom}} \rightarrow J^{3}(X \times \widetilde{C})_{\mathrm{tr}} .
$$

The following is well-known, see for example [Voi99].

Lemma 5.4. The transcendental Abel-Jacobi map AJ tr factorizes naturally

$$
\mathrm{AJ}_{\mathrm{tr}}: \mathrm{CH}^{2}(X \times \widetilde{C})_{\mathrm{hom}} /\left(\operatorname{Pic}(X) \times \mathrm{CH}^{1}(\widetilde{C})_{\mathrm{hom}}\right) \rightarrow J^{3}(X \times \widetilde{C})_{\mathrm{tr}} .
$$

In particular, $\mathrm{AJ}_{\mathrm{tr}}\left(Z_{C}\right)$ is independent of the choice of $L_{0}=\mathcal{O}\left(\sum n_{i} \cdot y_{i}\right) \in \operatorname{Pic}^{1}(\widetilde{C})$ as in (5.1).

Proof. One has to show that $\int_{\Gamma}$ is trivial on $T(X) \otimes H^{1}(\widetilde{C}, \mathbb{C})$ whenever $\partial \Gamma$ is of the form $D \times \sum n_{i} \cdot x_{i}$ for some curve $D \subset X$ and with $x_{i} \in \widetilde{C}$ and $\sum n_{i}=0$. But in this case one can assume that $\Gamma$ is of the form $D \times \Gamma_{0}$ with a path $\Gamma_{0} \subset \widetilde{C}$ and clearly $\int_{D}=0$ on $T(X)$.

The Abel-Jacobi class of $\mathrm{AJ}\left(\kappa_{C}\right)$ is the class $e_{X, C}$ in [Gre98], which can also be understood as an extension class in the category of mixed Hodge structures. The transcendental part is (essentially) the class studied further in [Voi99]. The second assertion of the following corollary has already been observed by Green in [Gre98].

Corollary 5.5. Suppose $C \subset X$ is an integral curve. Then

$$
\operatorname{AJ}_{\mathrm{tr}}\left(\kappa_{C}\right):=\operatorname{AJ}_{\mathrm{tr}}\left(Z_{C}\right) \in J^{3}(X \times \widetilde{C})_{\mathrm{tr}}
$$

is well defined. If $C$ is a constant cycle curve of order $n$, then $n \cdot \mathrm{AJ}_{\operatorname{tr}}\left(\kappa_{C}\right)=0$.

Proof. One argues as in the proof of Proposition 3.7. Using (3.8) and Lemma 5.4, one finds that $\mathrm{AJ}_{\mathrm{tr}}\left(Z_{C}\right)$ depends only on its restriction to some open subset $U:=\widetilde{C} \backslash\left\{p_{1}, \ldots, p_{k}\right)$. But if $n \cdot \kappa_{C}=0$, then $U$ can be chosen such that $n \cdot\left[\left.Z_{C}\right|_{X \times U}\right]=0$ in $\mathrm{CH}^{2}(X \times U)$ and hence $n \cdot \mathrm{AJ}_{\mathrm{tr}}\left(Z_{C}\right)=0$.

One can also project $\mathrm{AJ}\left(Z_{C}\right)$ onto a class $\mathrm{AJ}_{\text {alg }}\left(Z_{C}\right)$ in the algebraic part of the intermediate Jacobian.

$$
J^{3}(X \times \widetilde{C})_{\mathrm{alg}}:=\frac{F^{2}\left(\mathrm{NS}(X) \otimes H^{1}(\widetilde{C}, \mathbb{C})\right)^{*}}{\operatorname{NS}(X) \otimes H^{1}(\widetilde{C}, \mathbb{Z})} \simeq \operatorname{Pic}^{0}(\widetilde{C})^{\rho(X)} .
$$

To simplify the notation we shall henceforth assume $\rho(X)=1$ (but see Remark 5.10), so that $J^{3}(X \times \widetilde{C})_{\mathrm{alg}}=F^{2}\left([C] \otimes H^{1}(\widetilde{C}, \mathbb{C})\right)^{*} /\left([C] \otimes H^{1}(\widetilde{C}, \mathbb{Z})\right) \simeq \operatorname{Pic}^{0}(\widetilde{C})$.

Lemma 5.6. For an integral curve $C \in|L|$ with $L . L=2 g-2$, one has

$$
\operatorname{AJ}_{\mathrm{alg}}\left(Z_{C}\right)=f_{C}^{*} L \otimes\left(L_{0}^{2 g-2}\right)^{*} \in \operatorname{Pic}^{0}(\widetilde{C}) .
$$

Thus, if $L_{0}$ is chosen such that $L_{0}^{2 g-2} \simeq f_{C}^{*} L$, then $\mathrm{AJ}_{\mathrm{alg}}\left(Z_{C}\right)=0$. Cf. [Gre98, p. 270].

Proof. This must be standard. As I was not able to find a reference, I will sketch the argument. Let us first explain how $\mathrm{AJ}_{\mathrm{alg}}\left(Z_{C}\right)$ changes with $L_{0}$. The difference of the cycles for two different $L_{0}$ and $L_{0}^{\prime}$ is a cycle of the form $C \times D$ with $D$ a degree zero cycle on $C$. Then for any one-form $\alpha$ on $C$, one finds $\mathrm{AJ}_{\mathrm{alg}}\left(Z_{C}-Z_{C}^{\prime}\right)(C \times \alpha)=\int_{C \times \delta}[C] \times \alpha=(2 g-2) \int_{\delta} \alpha$, where $\partial \delta=D$. Hence, as predicted by the assertion, $\mathrm{AJ}_{\mathrm{alg}}\left(Z_{C}-Z_{C}^{\prime}\right)=\mathcal{O}((2 g-2) \cdot D) \simeq\left(L_{0}^{\prime} \otimes L_{0}^{*}\right)^{2 g-2}$. In particular, this allows us to restrict ourselves to the case $L_{0}=\mathcal{O}\left(y_{0}\right)$ for some point $y_{0} \in C$. 


\section{Curves AND CyCles ON K3 SuRfaCes}

Now let $\gamma \subset X \times C$ with $\partial \gamma=Z_{C}$. Then $\int_{\gamma}[C] \times \alpha=\int_{\gamma \cap\left(C^{\prime} \times C\right)} \operatorname{pr}_{2}^{*} \alpha$, where the deformation $C^{\prime}$ of $C$ is chosen generic such that $\gamma \cap\left(C^{\prime} \times C\right)$ is indeed one-dimensional. Note that $C^{\prime} \cap C:=$ $\left\{x_{1}, \ldots, x_{2 g-2}\right\}$ is a divisor in $\left.L\right|_{C}$. The intersection of $C^{\prime} \times C$ with $Z_{C}$ consists of the points $\left(x_{i}, x_{i}\right)$ and (with negative sign) $\left(x_{i}, y_{0}\right)$ and the intersection of $C^{\prime} \times C$ with $\gamma$ consists of paths connecting these points. Now project onto the second factor, which gives paths connecting the points $x_{i}=\operatorname{pr}_{2}\left(x_{i}, x_{i}\right)$ with $y_{0}=\operatorname{pr}_{2}\left(x_{i}, y_{0}\right)$, that is, a path with boundary $\left.L\right|_{C} \otimes \mathcal{O}\left((2 g-2) \cdot y_{0}\right)^{*}$. (Alternatively, one can pull back $\alpha$.)

The natural inclusion $T(X) \oplus \mathrm{NS}(X) \subset H^{2}(X, \mathbb{Z})$ is of finite index. Hence,

$$
J^{3}(X \times C) \rightarrow J^{3}(X \times \widetilde{C})_{\mathrm{tr}} \times J^{3}(X \times \widetilde{C})_{\mathrm{alg}}
$$

is finite of degree say $N$ (which depends only on $[C] \in H^{2}(X, \mathbb{Z}$ ) and not on $C$ ). In particular, if both, $\operatorname{AJ}\left(Z_{C}\right)_{\operatorname{tr}} \in J^{3}(X \times \widetilde{C})_{\operatorname{tr}}$ and $\operatorname{AJ}\left(Z_{C}\right)_{\text {alg }} \in J^{3}(X \times \widetilde{C})_{\text {alg }}$ vanish, then we also have $N \cdot \operatorname{AJ}\left(Z_{C}\right) \in J^{3}(X \times \widetilde{C})$.

5.3. It is difficult to decide for any given curve $C \subset X$ whether $\mathrm{AJ}_{\mathrm{tr}}\left(\kappa_{C}\right)$ is nontrivial. However, when put in a family, the resulting normal function is easier to control and from its nonvanishing one deduces the non-vanishing of $\mathrm{AJ}_{\mathrm{tr}}\left(\kappa_{C}\right)$ at least for generic $C$. This type of argument is standard (for hyperplane sections), but there are technical details that have to be adjusted to our situation. The key point is eventually the algebraicity of the zero-locus of normal functions recently established by Brosnan-Pearlstein [BP09] and M. Saito [Sai08], see [Cha13] for a recent overview.

We start with a positive-dimensional family of curves $T \subset|L|$ in a fixed linear system $|L|$ on $X$ with $L . L=2 g-2$. We assume that all curves $C \in T_{0}$ parametrized by a dense open subset $T_{0} \subset T$ are integral with (analytically) constant singularity type. Under this assumption, simultaneous normalization and then compactification (for both, one may need to replace the inclusion $T_{0} \subset|L|$ by a generically finite map $\left.T_{0} \longrightarrow|L|\right)$ yields a diagram

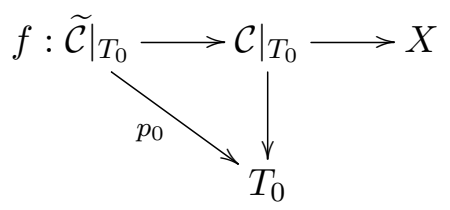

with $f$ dominant and such that $p_{0}:\left.\widetilde{\mathcal{C}}\right|_{T_{Q}} \rightarrow T_{0}$ is smooth with fibres given by the normalizations of the curves $\mathcal{C}_{t}, t \in T_{0}$. So, $f_{t}: \mathcal{C}_{t} \rightarrow \mathcal{C}_{t} \subset X$ for $t \in T_{0}$ is exactly as in the situation $f_{C}: \widetilde{C} \rightarrow C \subset X$ considered before.

Recall that the class of the cycle $Z_{C}$ on $X \times \widetilde{C}$ defined in (5.1) depends on the choice of a line bundle $L_{0}$ of degree one on $\widetilde{C}$. In order to define a global cycle that restricts to $Z_{C}$ on $X \times \widetilde{C}$ for a fibre $C=\mathcal{C}_{t}, t \in T_{0}$, we take the pull-back of $\left.\mathcal{C}\right|_{T_{0}} \rightarrow T_{0}$ to the subscheme $T_{0}^{\prime} \subset \operatorname{Pic}^{1}\left(\widetilde{\mathcal{C}} / T_{0}\right)$ of all line bundles $L_{0}$ on fibres $\widetilde{C}$ of $\left.\widetilde{\mathcal{C}}\right|_{T_{0}} \rightarrow T_{0}$ such that $L_{0}^{2 g-2} \simeq f_{C}^{*} L$. Replacing $T_{0}$ by (some further étale cover of) $T_{0}^{\prime}$, we may assume that $\left.\widetilde{\mathcal{C}}\right|_{T_{0}} \rightarrow T_{0}$ comes with a line bundle $\mathcal{L}_{0}$ on $\left.\widetilde{\mathcal{C}}\right|_{T_{0}}$ of degree one on all fibres $\widetilde{C}$ and such that $\left.\mathcal{L}_{0}^{2 g-2}\right|_{\widetilde{C}} \simeq f_{C}^{*} L$. Once this is achieved, one can compactify $\left.\left.\widetilde{\mathcal{C}}\right|_{T_{0}} \rightarrow \mathcal{C}\right|_{T_{0}} \rightarrow T_{0}$ to projective families $p: \widetilde{\mathcal{C}} \rightarrow \mathcal{C} \rightarrow T$ which still come with a morphism $f: \widetilde{\mathcal{C}} \rightarrow \mathcal{C} \rightarrow X$.

Next, consider the closure of the cycle

$$
Z_{\mathcal{C}}:=\Delta_{f}-\left.\mathcal{C}\right|_{T_{0}} \times_{T}\left[\mathcal{L}_{0}\right]-\left\{x_{0}\right\} \times\left.\widetilde{\mathcal{C}}\right|_{T_{0}}
$$




\section{Huybrechts}

on $X \times \widetilde{\mathcal{C}}$. Here, $\Delta_{f}$ is the pull-back of $\Delta_{X} \subset X \times X$ under id $\times f: X \times \widetilde{\mathcal{C}} \rightarrow X \times X$. Restricted to $X \times \widetilde{C}$ for $C=\mathcal{C}_{t}, t \in T_{0}$, the cycle $Z_{\mathcal{C}}$ yields $Z_{C}=\Delta_{f_{C}}-C \times\left[L_{0}\right]-\left\{x_{0}\right\} \times \widetilde{C}$ with $\left.L_{0} \simeq \mathcal{L}_{0}\right|_{\widetilde{C}}$.

We shall be interested in the normal function associated with this cycle. Denote by

$$
J^{3}:=J^{3}\left(X \times \widetilde{\mathcal{C}} / T_{0}\right) \rightarrow T_{0}
$$

the family of intermediate Jacobians of the family

$$
\pi:=p_{0} \circ p r_{2}: X \times\left.\widetilde{\mathcal{C}}\right|_{T_{0}} \longrightarrow T_{0}
$$

with fibres $X \times \widetilde{\mathcal{C}}_{t}$. Analogously, $J_{\text {tr }}^{3}$ and $J_{\text {alg }}^{3}$ denote the transcendental and algebraic parts with fibres over $t \in T_{0}$ as described by (5.3) and (5.5), respectively. In particular,

$$
J_{\text {alg }}^{3} \simeq \operatorname{Pic}^{0}\left(\widetilde{\mathcal{C}} / T_{0}\right) \rightarrow T_{0} .
$$

The sheaf of sections of $J^{3}$, denoted by the same symbol, is part of the short exact sequence

$$
0 \longrightarrow R^{3} \pi_{*} \mathbb{Z} \longrightarrow \mathcal{H}^{3} / F^{2} \mathcal{H}^{3} \longrightarrow J^{3} \longrightarrow 0,
$$

where $\mathcal{H}^{3}:=R^{3} \pi_{*} \Omega_{X \times \widetilde{\mathcal{C}} / T_{0}}^{\bullet}$ and $F^{2} \mathcal{H}^{3}:=R^{3} \pi_{*} \Omega_{X \times \widetilde{\mathcal{C}} / T_{0}}^{\geqslant 2}$.

Now, the fibrewise Abel-Jacobi classes $\operatorname{AJ}\left(Z_{\mathcal{C}_{t}}\right)$ induced by the global cycle $Z_{\mathcal{C}}$ yield the normal function

$$
\nu \in \Gamma\left(T_{0}, J^{3}\right)
$$

that is, a holomorphic section of $J^{3} \rightarrow T_{0}$, cf. [Voi02]. Similarly, its projections under $J^{3} \rightarrow J_{\mathrm{tr}}^{3}$ and $J^{3} \rightarrow J_{\text {alg }}^{3}$ are denoted by

$$
\nu_{\text {tr }} \in \Gamma\left(T_{0}, J_{\text {tr }}^{3}\right) \text { and } \nu_{\text {alg }} \in \Gamma\left(T_{0}, J_{\text {alg }}^{3}\right), \text { respectively. }
$$

Corollary 5.7. Under the above choice of $Z_{\mathcal{C}}$ and assuming $\rho(X)=1$, the algebraic part

$$
\nu_{\text {alg }} \in \Gamma\left(T_{0}, J_{\text {alg }}^{3}\right)=\Gamma\left(T_{0}, \operatorname{Pic}^{0}\left(\widetilde{\mathcal{C}} / T_{0}\right)\right)
$$

is trivial. Moreover, for the zero sets of the normal functions and any $n>0$, one has

$$
Z\left(n \cdot \nu_{\mathrm{tr}}\right) \subset Z(n \cdot N \cdot \nu),
$$

where $N$ is the degree of (5.6).

Proof. The first part is an immediate consequence of Lemma 5.6. For the second, recall that $n \cdot \mathrm{AJ}_{\mathrm{tr}}\left(Z_{C}\right)=0$ and $n \cdot \mathrm{AJ}_{\mathrm{alg}}\left(Z_{C}\right)=0$ imply $N \cdot n \cdot \mathrm{AJ}\left(Z_{C}\right)=0$.

Clearly, for $t \in T_{0}$ not contained in the zero locus of $Z(N \cdot \nu)$, the Abel-Jacobi class $N \cdot \operatorname{AJ}\left(Z_{C}\right)$ of $C:=\mathcal{C}_{t}$ and hence also $N \cdot \mathrm{AJ}_{\mathrm{tr}}\left(Z_{C}\right)$, are nontrivial. In order to prove $N \cdot \nu \neq 0$ and thus $T_{0} \backslash Z(N \cdot \nu) \neq \emptyset$, one describes its image under the boundary map of (5.8),

$$
\delta: \Gamma\left(T_{0}, J^{3}\right) \longrightarrow H^{1}\left(T_{0}, R^{3} \pi_{*} \mathbb{Z}\right),
$$

and shows that it is actually nontrivial. Note that $R^{3} \pi_{*} \mathbb{Z}=H^{2}(X, \mathbb{Z}) \otimes R^{1} p_{0 *} \mathbb{Z}$.

LEMma 5.8. The boundary class $\delta(\nu) \in H^{1}\left(T_{0}, R^{3} \pi_{*} \mathbb{Z}\right)$ is non-torsion.

Proof. We can restrict ourselves to the case $\operatorname{dim}(T)=1$.

- One first proves that $\left[Z_{\mathcal{C}}\right]$ is a nontrivial class in $[C]^{\perp} \otimes H^{2}\left(\left.\widetilde{\mathcal{C}}\right|_{T_{0}}, \mathbb{Z}\right) \subset H^{4}\left(X \times\left.\widetilde{\mathcal{C}}\right|_{T_{0}}, \mathbb{Z}\right)$.

Note that the cohomology class $(2 g-2) \cdot\left[Z_{\mathcal{C}}\right] \in H^{2}(X, \mathbb{Z}) \otimes H^{2}(\widetilde{\mathcal{C}}, \mathbb{Z})$ is the pull-back of $(2 g-$ $2) \cdot\left[\Delta_{X}\right]-[C] \times[C] \in H^{2}(X, \mathbb{Z}) \otimes H^{2}(X, \mathbb{Z})$, which is in fact the nontrivial class $(2 g-2) \cdot \mathrm{id} \in$ $[C]^{\perp} \otimes[C]^{\perp}$ (cf. Remark 5.3). (The argument is easily adapted to the case $g=1$.) Since $\widetilde{\mathcal{C}} \longrightarrow X$ is 


\section{Curves and CyCles on K3 surfaces}

dominant, the pull-back $T(X) \subset[C]^{\perp \subset} \longrightarrow H^{2}(\widetilde{\mathcal{C}}, \mathbb{Z})$ is injective. The kernel of $H^{2}(\widetilde{\mathcal{C}}) \longrightarrow H^{2}\left(\left.\widetilde{\mathcal{C}}\right|_{T_{0}}\right)$ is spanned by the divisor classes of the boundary $\left.\widetilde{\mathcal{C}} \backslash \widetilde{\mathcal{C}}\right|_{T_{0}}$ and, as $T(X)$ is an irreducible Hodge structure of level two, intersects $T(X)$ trivially.

- The further restriction to the fibres $\widetilde{\mathcal{C}_{t}}, t \in T_{0}$, is trivial, that is, under

$$
[C]^{\perp} \otimes H^{2}\left(\left.\widetilde{\mathcal{C}}\right|_{T_{0}}, \mathbb{Z}\right) \subset H^{4}\left(X \times\left.\widetilde{\mathcal{C}}\right|_{T_{0}}, \mathbb{Z}\right) \rightarrow H^{0}\left(T_{0}, R^{4} \pi_{*} \mathbb{Z}\right)
$$

the class $\left[Z_{\mathcal{C}}\right]$ vanishes. This is just rephrasing that the $Z_{C}$ are cohomologically trivial cycles.

- The Leray spectral sequence for $\pi: X \times\left.\widetilde{\mathcal{C}}\right|_{T_{0}} \rightarrow T_{0}$ yields a natural (surjective) map

$$
d: \operatorname{Ker}\left(H^{4}\left(X \times\left.\widetilde{\mathcal{C}}\right|_{T_{0}}, \mathbb{Z}\right) \rightarrow H^{0}\left(T_{0}, R^{4} \pi_{*} \mathbb{Z}\right)\right) \rightarrow H^{1}\left(T_{0}, R^{3} \pi_{*} \mathbb{Z}\right),
$$

the kernel of which is a quotient of the trivial group

$$
H^{2}\left(T_{0}, R^{2} \pi_{*} \mathbb{Z}\right)=\left(H^{2}(X, \mathbb{Z}) \otimes H^{2}\left(T_{0}, p_{0 *} \mathbb{Z}\right)\right) \oplus\left(H^{0}(X, \mathbb{Z}) \otimes H^{2}\left(T_{0}, R^{2} p_{0 *} \mathbb{Z}\right)\right)=0 .
$$

Here one uses that for dimension reasons all $H^{i}\left(T_{0}, R^{j} \pi_{*} \mathbb{Z}\right)$ are trivial for $i>2$ and also $H^{2}\left(T_{0}, p_{0 *} \mathbb{Z}\right) \simeq H^{2}\left(T_{0}, R^{2} p_{0 *} \mathbb{Z}\right)=0$ (after shrinking $T_{0}$, if necessary).

This yields a nontrivial class

$$
d\left[Z_{\mathcal{C}}\right] \in H^{1}\left(T_{0}, R^{3} \pi_{*} \mathbb{Z}\right)
$$

as the image of $\left[Z_{\mathcal{C}}\right]$. Since all the arguments above apply as well to multiples $N \cdot Z_{\mathcal{C}}$, the class $d\left[Z_{\mathcal{C}}\right]$ is in fact nontorsion.

- Eventually, one uses the fact that the class $d\left[Z_{\mathcal{C}}\right]$ is indeed $\delta\left(\nu_{Z}\right)$. See [Voi02].

Corollary 5.9. Under the above assumptions, there exist at most finitely many points $t \in T_{0}$ with $\mathrm{AJ}_{\mathrm{tr}}\left(Z_{\mathcal{C}_{t}}\right)=0$.

Proof. Here one uses that the vanishing locus $Z(N \cdot \nu) \subset T_{0}$ of the normal function $N \cdot \nu \in$ $\Gamma\left(T_{0}, J^{3}\right)$ is an algebraic set due to [BP09, Sai08]. Thus, if $Z(N \cdot \nu)$ is zero-dimensional, then it can only be a finite set of points and hence $Z\left(\nu_{\text {tr }}\right)$ is also finite by Corollary 5.7. If $Z(N \cdot \nu)$ has positive dimensional components, then repeat the above argument with $T_{0}$ replaced by such a component. But over the new $T_{0}$, the class $N \cdot \delta\left[Z_{\mathcal{C}}\right]$ would be trivial, contradicting that $\delta\left[Z_{\mathcal{C}}\right]$ is nontorsion by Lemma 5.8.

Remark 5.10. For simplicity we assumed that $\rho(X)=1$ in the above discussion. The case $\rho(X)>1$ is dealt with similarly, either by replacing $H^{2}(X, \mathbb{Z})$ throughout by $T(X) \oplus \mathbb{Z} \cdot[C]$, by observing that the arguments above prove directly that $\nu_{\mathrm{tr}} \neq 0$ (without controlling the algebraic part) or by working with the cycle $Z_{X}$ in Remark 5.3 (i).

5.4. To conclude the proof of Proposition 5.1, one stratifies $|L|$ according to the singularity types of the curve and their number of integral components. Since eventually there are only finitely many strata and for each stratum finiteness of curves $C:=\mathcal{C}_{t}$ with $n \cdot \operatorname{AJ}_{\operatorname{tr}}\left(\kappa_{C}\right)=0$ is assured by Corollary 5.9, the proposition then follows from Corollary 5.5.

5.5. Suppose $\mathcal{C} \subset \mathcal{X} \rightarrow T$ is a flat family of curves $\mathcal{C}_{t}$ in $\mathrm{K} 3$ surfaces $\mathcal{X}_{t}$. Standard arguments prove that the locus of $t \in T$ for which $\mathcal{C}_{t} \subset \mathcal{X}_{t}$ is a constant cycle curve is a countable union of Zariski closed subsets of $T$. Indeed, by using that the (relative) Hilbert scheme (of the relative symmetric products) is a countable union of projective schemes over $T$, one proves that the set 


\section{Huybrechts}

of points $x \in \mathcal{C}_{t}$ with $[x]=c_{\mathcal{X}_{t}}$ is a countable union of Zariski closed subsets (see for example [Voi02, Ch. 22.1]).

Without bounding the order, the result cannot be improved (cf. Lemma 6.5). For the trivial family $\mathcal{X}=X \times T$, Proposition 5.1 proves that the locus of $t \in T$ with $\mathcal{C}_{t} \subset X$ a constant cycle curve of order $\leqslant n$ is Zariski closed. However, the Hodge theoretic line of reasoning seems not to extend to nontrivial families $\mathcal{X} \longrightarrow T$. Only the locus of $t \in T$ with $n \cdot \mathrm{AJ}_{\mathrm{tr}}\left(\kappa_{\mathcal{C}_{t}}\right)=0$ describes a Zariski closed subset.

\section{First examples of constant cycle curves}

It is notoriously difficult to construct rational curves on K3 surfaces, see Section 2.1. As constant cycle curves are natural generalizations of rational curves, in particular with respect to (2.3), it seems worthwhile to work out examples. On the one hand, it will become clear that constant cycle curves are much easier to construct than rational curves, at least if we do not care about their order. On the other hand, the powerful technique developed in [BHT11, LL12] to prove the existence of rational curves by reducing modulo $p$ and then using Tate's conjecture does not seem to apply to constant cycle curves. The main reason being that a curve could be a constant cycle curve modulo infinitely many primes without being a constant cycle curve itself. In fact, all curves over $\overline{\mathbb{F}}_{p}$ should be constant cycle curves (see Section 9 ), but not over $\overline{\mathbb{Q}}$.

If not stated otherwise, $X$ will be a K3 surface over an arbitrary algebraically closed field $k$.

6.1. As mentioned before, all rational curves $C \subset X$ are pointwise constant cycle curves. In fact, we have the following result.

Lemma 6.1. A rational curve $C \subset X$ in a $K 3$ surface is a constant cycle curve of order one.

Proof. This is easy to see by using (3.8), which together with $\mathrm{CH}^{2}\left(X \times \mathbb{P}^{1}\right) \simeq \mathrm{CH}^{2}(X) \oplus \mathrm{CH}^{1}(X)$. $h$, where $h$ is the hyperplane section on $\mathbb{P}^{1}$, shows that $\mathrm{CH}^{2}(X) \simeq \mathrm{CH}^{2}(X \times U)$ for every proper nonempty open subset $U \subset \mathbb{P}^{1}$. Hence, $\mathrm{CH}^{2}\left(X \times k\left(\eta_{C}\right)\right) \simeq \mathrm{CH}^{2}(X)$, which is torsion free.

6.2. We shall discuss a construction inspired by [Voi12b]. In particular, as remarked in [Voi12b, Lem. 2.3], it shows that the union of all constant cycle curves is dense (even in the classical topology for complex K3 surfaces).

Consider an elliptic K3 surface with a zero-section

$$
\pi: X \rightarrow \mathbb{P}^{1}, C_{0} \subset X .
$$

We first give an ad hoc and geometric description of the constant cycle curves of torsion points on the fibres. For this assume that $k$ is uncountable. The formal definition for an arbitrary algebraically closed field $k$, which is also needed to determine the order of $C_{n}$, is given below. Let

$$
C_{n} \subset X
$$

be the closure of the set of $n$-torsion points on the smooth fibres $X_{t}, t \in \mathbb{P}^{1}$. Let $x_{t} \in X_{t}$ denote the origin, that is, $\left\{x_{t}\right\}=C_{0} \cap X_{t}$. Thus, for any $x \in C_{n} \cap X_{t}$, the class $[x]-\left[x_{t}\right]$ is $n$-torsion in $\mathrm{CH}^{1}\left(X_{t}\right)$ and hence trivial in $\mathrm{CH}^{2}(X)$. Hence, $C_{n}$ is a pointwise constant cycle curve and by Proposition 3.7 in fact a constant cycle curve.

For arbitrary $k$ (as always algebraically closed), these curves (or rather their irreducible components) are constructed as follows: Denote by $\mu \in \mathbb{P}^{1}$ the generic point and by $X_{\mu}$ the 


\section{CuRves AND CYCLES ON K3 SURFACES}

generic fibre of $\pi$. For $x \in X_{\mu}$ with $k(x) / k(\mu)$ finite, let $C_{x} \subset X$ be the curve obtained as the closure of the point $x \in X_{\mu} \subset X$. In particular, the generic point $\eta_{C_{x}} \in C_{x}$ is just $x \in X_{\mu}$ and $k\left(\eta_{C_{x}}\right)=k(x)$. The diagonal of $C_{x}$ as a subvariety $\Delta_{C_{x}} \subset X \times C_{x}$ is in fact contained in the surface $X \times \times_{\mathbb{P}^{1}} C_{x}$. Hence, its class $\left[\Delta_{C_{x}}\right] \in \mathrm{CH}^{2}\left(X \times C_{x}\right)$ is the push-forward under

$$
\mathrm{CH}^{1}\left(X \times \mathbb{P}^{1} C_{x}\right) \rightarrow \mathrm{CH}^{2}\left(X \times C_{x}\right)
$$

of the class of the relative diagonal $\Delta_{C_{x} / \mathbb{P}^{1}} \subset X \times_{\mathbb{P}^{1}} C_{x}$. Restricting to the generic point $\eta_{C_{x}}$ shows that $\left[\left.\Delta_{C_{x}}\right|_{X \times k\left(\eta_{C_{x}}\right)}\right] \in \mathrm{CH}^{2}\left(X \times k\left(\eta_{C_{x}}\right)\right)$ is the push-forward of the class of the point $x \in X_{\mu}$ in $\mathrm{CH}^{1}\left(X \times_{\mathbb{P}^{1}} k(x)\right)=\mathrm{CH}^{1}\left(X_{\mu} \times_{k(\mu)} k(x)\right)$.

This observation can now be applied to the origin $x=o \in X_{\mu}$, that is, the intersection of $C_{0}$ with $X_{\mu}$, and to any point $x_{n} \in X_{\mu}$ of order $n$. For the latter, the associated curve

$$
C_{x_{n}}:=\overline{\left\{x_{n}\right\}} \subset X
$$

is an irreducible component of the curve $C_{n}$ of $n$-torsion points in the fibres as considered above.

Lemma 6.2. (i) The curve $C_{x_{n}}$ is a constant cycle curve of order $d \mid n$.

(ii) The union of all $C_{x_{n}}$ is dense and if $k=\mathbb{C}$ even dense in the classical topology.

Proof. The density statement (ii) is obvious, as the set of geometric torsion points is dense in the generic fibre $X_{\mu}$.

For statement (i), first note that $\left[\Delta_{C_{0}}\right] \in \mathrm{CH}^{2}\left(X \times C_{0}\right)=\mathrm{CH}^{2}\left(X \times \mathbb{P}^{1}\right)$ is the class of $\left\{x_{0}\right\} \times C_{0}+C_{0} \times\left\{x_{0}\right\}$ (for an arbitrary point $x_{0} \in C_{0}$ ), which restricted to $X \times k(o)$ is $\left[x_{0}\right] \times k(o)$. Thus, the restriction of the class of $\Delta_{C_{x_{n}}}-\left\{x_{0}\right\} \times C_{x_{n}}$ to $X \times k\left(\eta_{C_{x_{n}}}\right)$, which by definition is $\kappa_{C_{x_{n}}}$, is the image of $\left[x_{n}-o\right] \in \mathrm{CH}^{1}\left(X_{\mu} \times_{k(\mu)} k\left(x_{n}\right)\right)$. Here, since $k(o)=k(\mu)$, the class $[o] \in \mathrm{CH}^{1}\left(X_{\mu}\right)$ can be base changed to a class in $\mathrm{CH}^{1}\left(X_{\mu} \times_{k(\mu)} k\left(x_{n}\right)\right)$.

As $x_{n} \in X_{\mu}$ is an $n$-torsion point, the class $\left[x_{n}-o\right]$ is $n$-torsion and so is its image $\kappa_{C_{x_{n}}}$.

Remark 6.3. Note that it could happen that $C_{x_{n}}$ is of order $d<n$, for example when $x_{n}$ is a $k(\mu)$-rational point and, therefore, that $C_{x_{n}}$ a rational curve. But presumably in the generic situation $\kappa_{C_{x_{n}}}$ is of order exactly $n$. In any case, this elementary construction already provides many examples of constant cycle curves which are not rational.

The construction will now be generalized to covering families of elliptic curves, still following [BV04, Voi12b]: First, the existence of nodal rational curves on the generic K3 surface leads to the existence of a dominating family of elliptic curves for every K3 surface. More precisely, for an arbitrary K3 surface $X$ there exists a smooth elliptic surface $\mathcal{C} \rightarrow T$ with a surjective morphism $p: \mathcal{C} \longrightarrow X$. See [HT00, Thm. 4.1] for details in the case of characteristic zero. In positive characteristic use a lift to characteristic zero and reduce the family of curves back to $p$, which yields a family of elliptic curves if $X$ is not unirational. The unirational case being trivial, we shall just ignore it. Note that then, one could moreover assume that the generic fibre $\mathcal{C}_{t}$ is mapped birationally onto its image in $X$, but we will not need this.

Now, choose an ample rational curve $C_{0} \subset X$ and let $\widetilde{C}_{0}$ be its preimage in $\mathcal{C}$. Replacing $\mathcal{C} \rightarrow T$ by its base change to $\widetilde{C}_{0}$, we can assume that $\mathcal{C} \rightarrow T$ admits a section $\sigma_{0}: T \rightarrow \mathcal{C}$ such that $\sigma_{0}(T)$ maps onto a rational curve in $X$, namely $C_{0}$. Then consider the curve $C_{n} \subset X$ defined as the closure of the set of images of all points $x \in \mathcal{C}_{t}$ in the smooth fibres $\mathcal{C}_{t}, t \in T$, such that $n \cdot\left([x]-\left[x_{t}\right]\right)=0$ in $\mathrm{CH}^{1}\left(\mathcal{C}_{t}\right)$, where $x_{t}$ is the point of intersection of $\mathcal{C}_{t}$ with $\sigma_{0}(T)$. For the same reason as before, the curve $C_{n}$ is a (possibly reducible) pointwise constant cycle curve. Note that, 


\section{Huybrechts}

indeed, any torsion point in a smooth fibre $\mathcal{C}_{t}$ is contained in a multi-section of $\mathcal{C} \longrightarrow T$ consisting of torsion points in the smooth fibres. Also, the arguments in the proof of Lemma 6.2 still apply.

The following was already explicitly stated in [Voi12b] and was also used in [Mac04]. It should be compared to Conjecture 3 .

Corollary 6.4. On any K3 surface $X$ over an arbitrary algebraically closed field $k$, the union

$$
\bigcup_{C=\mathrm{ccc}} C \subset X
$$

of constant cycle curves (of unbounded order) is dense. For $k=\mathbb{C}$ density holds in the classical topology.

6.3. In Section 6.2 we explained how to produce constant cycle curves as multi-sections of elliptic fibrations. But, as was pointed out by Claire Voisin, smooth (and hence non-rational) fibres can also be constant cycle curves.

Consider the Kummer surface $X$ obtained as the minimal resolution of the standard involution on the product $E_{1} \times E_{2}$ of two elliptic curves. Let

$$
X \rightarrow\left(E_{1} \times E_{2}\right) / \pm \rightarrow \mathbb{P}^{1} \simeq E_{1} / \pm
$$

be the elliptic fibration induced by the first projection. There are only four singular fibres, all of type $I_{0}^{*}$ over the two-torsion points. Being rational, they are constant cycle curves of order one.

Now consider a torsion point $t \in E_{1}$ of order $n \neq 2$ and the fibre $C_{t} \subset X$ over $\bar{t} \in \mathbb{P}^{1}$.

Lemma 6.5. For a torsion point $t \in E_{1}$ of order $n \neq 2$, the associated fibre $C_{t} \subset X$ is a smooth constant cycle curve of order $d \mid n$.

Proof. Indeed, if $C_{t} \subset X$ is viewed with respect to the other elliptic fibration $X \rightarrow \mathbb{P}^{1} \simeq E_{2} / \pm$, then it intersects the (smooth) fibres in $n$-torsion points. In particular, it intersects the generic fibre in an $n$-torsion point $x_{n} \in E_{1} \times K\left(E_{2} / \pm\right)$. Thus, $C_{t}$ is one of the curves $C_{x_{n}}$ considered in Lemma 6.2 and hence a constant cycle curve of order $d \mid n$. (It seems likely that the order equals $n$, but for proving this one would need to control the kernel of $\mathrm{CH}^{1}\left(X_{\mu}\right) \rightarrow \mathrm{CH}^{2}\left(X \times_{k} k(\mu)\right)$, see Section 6.2.)

In particular, this construction yields an elliptic K3 surface $X \rightarrow \mathbb{P}^{1}$ with infinitely many fibres that are constant cycle curves (of growing order). It is not clear to me whether this holds for arbitrary elliptic K3 surfaces. Also note that a smooth fibre of an elliptic K3 surface $X \rightarrow \mathbb{P}^{1}$ can even be a constant cycle curve of order one, see Example 7.3 and the discussion in Section 10.2. The above construction dispels hope expressed in [Ker08, Sec. 17] that the Abel-Jacobi class of a smooth fibre should in particular always be nontorsion.

\section{More examples: fixed curves}

We start this section with the branching curve of a double plane which turns out to be a constant cycle curve of order at most two. This then generalizes to fixed point curves of arbitrary nonsymplectic automorphisms. For simplicity we work in characteristic zero.

7.1. Consider a generic double plane, that is, K3 surface $X$ given as a $2: 1$ cover

$$
X \rightarrow \mathbb{P}^{2}, \quad i: X \stackrel{\sim}{\longrightarrow} X
$$




\section{CuRves AND CYCLES ON K3 SURFACES}

ramified over a smooth curve $C \subset \mathbb{P}^{2}$ of degree six with $i$ the covering involution. Using the eigenspace decomposition one finds that $i^{*}=-\mathrm{id}$ on $\mathrm{CH}^{2}(X)_{0}$, for $\mathrm{CH}^{2}\left(\mathbb{P}^{2}\right)_{0}=0$.

Now consider $C$ as a curve in $X$ and write the class $[x]$ of a point $x \in C$ as $[x]=c_{X}+\alpha_{x}$ with $\alpha_{x} \in \mathrm{CH}^{2}(X)_{0}$. On the one hand, $i^{*}[x]=[i(x)]=[x]$ and on the other hand, $i^{*}[x]=$ $i^{*}\left(c_{X}+\alpha_{x}\right)=c_{X}-\alpha_{x}$. Hence, for $x \in C$, one has $2 \cdot \alpha_{x}=0$ and, since $\mathrm{CH}^{2}(X)$ is torsion free, we also have $\alpha_{x}=0$, that is, $C$ is a (pointwise) constant cycle curve. This provides an explicit example of a constant cycle curve which is smooth and of genus ten (and so in particular not rational). As we shall see in broader generality, $C$ is a constant cycle curve of order one or two. This already shows that in general, the genus of a constant cycle curve is not determined by its order. Finding an example of a constant cycle curve of order one that is nonrational is harder, see Corollary 7.2.

7.2. This naive example is now generalized as follows. Suppose $f: X \stackrel{\sim}{\longrightarrow} X$ is an automorphism of finite order $n$. Assume that the quotient

$$
\pi: X \rightarrow \bar{X}:=X /\langle f\rangle,
$$

which is possibly singular, satisfies $\mathrm{CH}^{2}(\bar{X})_{0}=0$. Note that due to Bloch's conjecture (cf. Section 4 ), which is known for surfaces of Kodaira dimension $<2$, the latter condition is equivalent to $f^{*} \neq$ id on $H^{2,0}(X)$. Suppose a curve $C \subset X$ is contained in the fixed point locus $\operatorname{Fix}(f)$. Then a similar trick as above shows that $C$ is a (pointwise) constant cycle curve. Indeed, write $[x]=c_{X}+\alpha_{x}$ for $x \in C$. Then $n \cdot[x]=n \cdot c_{X}+n \cdot \alpha_{x}$, but on the other hand $n \cdot[x]$ is the pull-back of $[\pi(x)] \in \mathrm{CH}^{2}(\bar{X})$. Hence, $n \cdot[x]=n \cdot c_{X}$, which yields $n \cdot \alpha_{x}=0$ and, therefore, $\alpha_{x}=0$. The calculation in this case suggests that any curve in the fixed point locus of a nonsymplectic automorphism of finite order $n$ is a constant cycle curve of order $d \mid n$. This can be shown rigorously as follows.

Proposition 7.1. Let $f: X \stackrel{\sim}{\rightarrow} X$ be an automorphism of finite order $n$ of a K3 surface $X$ over an algebraically closed field $k$ of $\operatorname{char}(k)=0$ such that $f^{*} \neq \mathrm{id}$ on $H^{0}\left(X, \Omega_{X}^{2}\right)$. Then any curve $C \subset X$ contained in $\operatorname{Fix}(f)$ is a constant cycle curve of order $d \mid n$.

Proof. Consider the pull-back $\pi^{*}: \mathrm{CH}^{2}(\bar{X}) \rightarrow \mathrm{CH}^{2}(X)$ induced by the projection $\pi: X \rightarrow \bar{X}$. Let $y:=\pi(x)$ for a fixed point $x \in X$. Then $\pi^{*}[y]=n \cdot c_{X}$ if $[x]=c_{X}$ and $\pi^{*}[y]=n \cdot[x]$. The same holds after base change to any field extension $K / k$. Apply this to $K=k\left(\eta_{C}\right)$ for a curve $C \subset X$ in the fixed point locus of $f$. Then the generic point $\eta_{C} \in C$, viewed as a closed point $\eta_{C} \in X \times k\left(\eta_{C}\right)$, is fixed under $f_{k\left(\eta_{C}\right)}$. Similarly, the generic point $\eta_{\bar{C}} \in \bar{C}:=\pi(C) \subset \bar{X}$ can be viewed as a closed point in $\bar{X} \times k\left(\eta_{\bar{C}}\right)$ and, moreover, $k\left(\eta_{\bar{C}}\right)=k\left(\eta_{C}\right)$. Then $\left[\eta_{\bar{C}}\right] \longmapsto n \cdot\left[\eta_{C}\right]$ under

$$
\pi_{k\left(\eta_{C}\right)}^{*}: \mathrm{CH}^{2}\left(\bar{X} \times k\left(\eta_{\bar{C}}\right)\right) \rightarrow \mathrm{CH}^{2}\left(X \times k\left(\eta_{C}\right)\right) .
$$

Since $f^{*} \neq$ id on $H^{0}\left(X, \Omega_{X}^{2}\right)$ and $\operatorname{kod}(\bar{X})<2$, Bloch's conjecture holds true for $\bar{X}$, that is, $\mathrm{CH}^{2}(\bar{X}) \simeq \mathbb{Z}$. In particular, there is a distinguished generator $c_{\bar{X}} \in \mathrm{CH}^{2}(\bar{X})$ with $\pi^{*}\left(c_{\bar{X}}\right)=n \cdot c_{X}$. In order to conclude, it is therefore enough to prove that $\left[\eta_{\bar{C}}\right] \in \mathrm{CH}^{2}\left(\bar{X} \times k\left(\eta_{\bar{C}}\right)\right)$ is in the image of the base change map $\mathrm{CH}^{2}(\bar{X}) \rightarrow \mathrm{CH}^{2}\left(\bar{X} \times k\left(\eta_{\bar{C}}\right)\right)$. Indeed, then the image $n \cdot \kappa_{C}$ of $\left[\eta_{\bar{C}}\right]-c_{\bar{X}} \times k\left(\eta_{\bar{C}}\right)=0$ under $(7.1)$ would also be zero.

In other words, it is enough to prove that any curve in $\bar{X}$ (and so in particular $\bar{C}$ ) is a constant cycle curve of order one. As by assumption $f$ has a fixed curve and hence $\bar{X}$ is rational, this follows from Proposition 4.2, ii). 


\section{Huybrechts}

7.3. Nonsymplectic automorphisms have been studied intensively in the literature. If the order is prime, only $p=2,3,5,7,11,13,17$, and 19 can occur. Their fixed point loci can be described, often in addition to isolated fixed points and rational curves they also contain smooth elliptic curves and even smooth curves of higher genus, see for example [AST11]. However, the genus of constant cycle curves obtained in this way is rather small, for example for $p=7,11$ at most elliptic curves can occur and for $p=13,17,19$ all curves in $\operatorname{Fix}(f)$ are in fact rational. The maximal genus $g=11$ can be achieved for $p=2$.

Corollary 7.2. Consider an automorphism $f \in \operatorname{Aut}(X)$ of order $p \cdot q$ for two primes $p \neq q$. Assume that $f^{p}$ and $f^{q}$ are both nonsymplectic, that is, $f^{*}$ acts by a primitive $(p \cdot q)$-th root of unity on $H^{0}\left(X, \Omega_{X}^{2}\right)$. Then any curve $C \subset X$ in $\operatorname{Fix}(f)$ is a constant cycle curve of order one.

Proof. Under the assumptions, the curve $C$ would be in the fixed point locus of both $f^{p}$ and $f^{q}$. Hence, $C$ is a constant cycle curve of order dividing $q$ and $p$ and, therefore, of order one.

Example 7.3. As observed by Dillies in [Di109] and extended by Garbagnati and Sarti in [GS10], nonsymplectic automorphisms of prime order $p$ often occur as the square $f^{2}$ of an automorphism $f$ with $f^{p}$ a nonsymplectic involution. The corollary applies in this situation to curves in $\operatorname{Fix}(f)$. Cases where a nonrational curve is contained in $\operatorname{Fix}(f)$ can be found in [Dil09, GS10].

It is worth pointing out that in the explicit example described in [Dil09, Sect. 7], the K3 surface $X$ comes with an elliptic fibration $\pi: X \rightarrow \mathbb{P}^{1}$ which is preserved by $f$ and such that one of the smooth(!) fibres is contained in $\operatorname{Fix}(f)$. Concretely, $X$ is the elliptic surface given by the Weierstrass equation $y^{2}=x^{3}+\left(t^{6}-1\right)^{2}$ and $f(x, y, t)=(x, y, \xi \cdot t)$ with $\xi$ a primitive sixth root of unity.

As Alessandra Sarti informed me, this example can be generalized to yield a whole family of elliptic K3 surfaces with a purely nonsymplectic automorphism of order six with a smooth elliptic curve in the fixed point locus, which is therefore a constant cycle curve of order one.

For another family of examples, see $X_{3,1}$ described in [AS08, Prop. 4.7]. It is not difficult to write down a square root of the order three automorphism given there, but the elliptic structure in the example is not obvious.

This then eventually yields an example of a constant cycle curve of order one which is not rational (but smooth elliptic).

COROLlary 7.4. There exist nonrational constant cycle curves of order one.

Although it might be difficult to exhibit explicitly constant cycle curves of order one and arbitrary high genus, there does not seem to be any reason why this should not be possible.

\section{Bitangent correspondence}

Here, we exhibit a more involved example, close in spirit to the ones described in Section 7 , which leads to constant cycle curves of order at most four and geometric genus 201. These curves will be constructed as the fixed locus of the 'bitangent correspondence' for a generic quartic K3 surface. The bitangent correspondence maps a generic point to the second contact point of a bitangent at $x$. Since generically there are six bitangents at every point, this does not define a map, but we will show that it is well defined on the Chow ring. Its fixed locus is the curve of contact points of hyperflexes. 


\section{Curves AND CyCles ON K3 SuRfaCes}

Recall that for a quartic $X \subset \mathbb{P}^{3}$, a line $\ell \subset \mathbb{P}^{3}$ is called a bitangent of $X$ if at every $x \in X \cap \ell$ the intersection multiplicity is at least two. A bitangent $\ell$ is a hyperflex if there is a unique point of intersection (and, clearly, the intersection multiplicity is then four).

In this section we shall work over $\mathbb{C}$.

8.1. Consider a smooth quartic $X \subset \mathbb{P}^{3}$ not containing a line. For generic $x \in X$, the curve

$$
C_{x}:=T_{x} X \cap X
$$

has exactly one singularity, a node at $x$. Let $\nu: \widetilde{C}_{x} \rightarrow C_{x}$ be its normalization and let $f: \widetilde{C}_{x} \rightarrow X$ be its composition with the inclusion $C_{x} \subset X$. Then $\widetilde{C}_{x}$ is a smooth curve of genus two.

Choose a generic line $\mathbb{P}^{1} \subset T_{x} X \simeq \mathbb{P}^{2}$ and consider the linear projection $\varphi: C_{x} \rightarrow \mathbb{P}^{1}$ from the node $x \in C_{x}$. As $\operatorname{deg}\left(C_{x}\right)=4$ and $x \in C_{x}$ is a node, $\varphi$ is of degree two and so is the composition $\tilde{\varphi}: \widetilde{C}_{x} \rightarrow \mathbb{P}^{1}$. By the Hurwitz formula, the ramification divisor of $\tilde{\varphi}$ is of degree six. Thus, for generic choices there are exactly six lines

$$
\ell_{1}, \ldots, \ell_{6} \subset T_{x} X
$$

passing through $x$ and bitangent to $C_{x}$ at some other point $y_{1}, \ldots, y_{6} \in C_{x}$. (This is classical and well known. All it says is that there are exactly six bitangents through a generic $x \in X$.)

The construction in particular shows that up to two-torsion, the points $y_{1}, \ldots, y_{6} \in \widetilde{C}_{x}$ are linearly equivalent, as $\tilde{\varphi}^{*} \mathcal{O}(1) \simeq \mathcal{O}\left(2 \cdot y_{i}\right)$ for all $i$. Thus, for $f_{*}: \operatorname{Pic}\left(\widetilde{C}_{x}\right) \longrightarrow \mathrm{CH}^{2}(X)$ one finds $f_{*} \mathcal{O}(1)=2 \cdot\left[y_{i}\right] \in \mathrm{CH}^{2}(X)$ and hence $2 \cdot\left[y_{1}\right]=\ldots=2 \cdot\left[y_{6}\right]$. Since $\mathrm{CH}^{2}(X)$ is torsion free, in fact $\left[y_{1}\right]=\ldots=\left[y_{6}\right] \in \mathrm{CH}^{2}(X)$.

So the $1: 6$ correspondence $x \longmapsto\left\{y_{1}, \ldots, y_{6}\right\}$ induces a well-defined involution(!)

$$
\gamma: \mathrm{CH}^{2}(X) \stackrel{\sim}{\longrightarrow} \mathrm{CH}^{2}(X), \quad[x] \longmapsto\left[y_{1}\right] .
$$

In fact, $6 \cdot \gamma=\left[\Gamma_{X}\right]_{*}$, where $\Gamma_{X}$ is the closure of the locus $\{(x, y) \mid x \neq y, \overline{x, y}$ bitangent $\} \subset X \times X$.

Note that for $(x, y) \in \Gamma_{X}$, so generically $\overline{x, y}$ is a bitangent, the class $\alpha:=[x]-[y]$ satisfies $\gamma(\alpha)=-\alpha$. This can be proved in general.

Proposition 8.1. The bitangent correspondence $\gamma$ acts by $-\mathrm{id}$ on $\mathrm{CH}^{2}(X)_{0}$.

Proof. Write $[x]=c_{X}+\alpha_{x}$ for any $x \in X$. We have to show that $\gamma\left(\alpha_{x}\right)=-\alpha_{x}$. Since any point is rationally equivalent to a cycle contained in a fixed nonempty open subset, we can assume that $x$ is generic as above. For $\tilde{\varphi}: \widetilde{C}_{x} \rightarrow \mathbb{P}^{1}$, the Hurwitz formula yields $\omega_{\widetilde{C}_{x}} \simeq \tilde{\varphi}^{*} \mathcal{O}_{\mathbb{P}^{1}}(-2) \otimes \mathcal{O}\left(\sum y_{i}\right)$. On the other hand, $\omega_{\widetilde{C}_{x}} \simeq \nu^{*} \omega_{C_{x}} \otimes \mathcal{O}\left(-x_{1}-x_{2}\right)$, where $x_{1}, x_{2} \in \widetilde{C}_{x}$ are the two points over the node $x \in C_{x}$. Since $\left.\omega_{C_{x}} \simeq \omega_{T_{x} X} \otimes \mathcal{O}_{T_{x} X}(4) \simeq \mathcal{O}_{\mathbb{P}^{3}}(1)\right|_{C_{x}}$, one obtains

$$
\mathcal{O}\left(\sum y_{i}\right) \simeq \nu^{*}\left(\left.\mathcal{O}_{\mathbb{P}^{3}}(1)\right|_{C_{x}}\right) \otimes \tilde{\varphi}^{*} \mathcal{O}_{\mathbb{P}^{1}}(2) \otimes \mathcal{O}\left(-x_{1}-x_{2}\right) \simeq \nu^{*}\left(\left.\mathcal{O}_{\mathbb{P}^{3}}(3)\right|_{C_{x}}\right) \otimes \mathcal{O}\left(-3 \cdot x_{1}-3 \cdot x_{2}\right),
$$

where one uses $\tilde{\varphi}^{*} \mathcal{O}_{\mathbb{P}^{1}}(1) \simeq \nu^{*}\left(\left.\mathcal{O}_{\mathbb{P}^{3}}(1)\right|_{C_{x}}\right) \otimes \mathcal{O}\left(-x_{1}-x_{2}\right)$.

But then (using (2.1)), we have

$$
\left[\Gamma_{X}\right]_{*}[x]=f_{*}\left(\sum y_{i}\right)=3 \cdot\left(h . C_{x}\right)-6 \cdot[x]=6 \cdot c_{X}-6 \cdot \alpha_{x} .
$$

For a point $x$ with $\alpha_{x}=0$ this shows that $\left[\Gamma_{X}\right]_{*} c_{X}=6 \cdot c_{X}$. Consequently, for arbitrary $x \in X$ we also have $\left[\Gamma_{X}\right]_{*} \alpha_{x}=-6 \cdot \alpha_{x}$.

Corollary 8.2. The bitangent correspondence $\gamma$ acts by -id on $H^{2,0}(X)$. 


\section{Huybrechts}

Proof. This follows from the 'easy direction' of the general conjectures on the Bloch-Beilinson filtration, see for example [Voi02, Prop. 23.18]. See also Remark 8.5.

8.2. There is a more geometric way of defining this correspondence. Consider the universal family of bitangents:

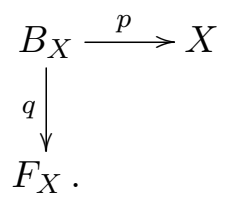

More explicitly, bitangents $\ell$ of $X$ correspond bijectively to points $[\ell] \in F_{X}$ and $B_{X}$ is the variety of all $(\ell, x)$, with $\ell$ a bitangent of $X$ and $x$ is a point of contact, that is, $x \in X \cap \ell$. By $p$ and $q$ we denote the projections $(\ell, x) \longmapsto x$ and $(\ell, x) \longmapsto[\ell]$, respectively. In particular, $q$ is of degree two with a well-studied ramification divisor $D_{\mathrm{hf}} \subset F_{X}$ (the curve of hyperflexes) and $p$ is of degree six, as there are exactly six bitangents at a generic $x$. As shown by Tikhomirov and Welters, $F_{X}$ and $B_{X}$ are smooth irreducible surfaces (of general type), see [Tik80, Wel81].

Now consider the covering involution

$$
i: B_{X} \rightarrow B_{X}
$$

of the double cover $q: B_{X} \rightarrow F_{X}$. So for generic bitangent $\ell$, the involution $i$ interchanges $(\ell, x)$ and $(\ell, y)$, where $X \cap \ell=\{x, y\}$.

Then $\Gamma_{X} \subset X \times X$ is the image of the natural morphism

$$
g: B_{X} \longrightarrow X \times X,(\ell, x) \longmapsto(x=p(\ell, x), p(i(\ell, x))) .
$$

In other words, $g(\ell, x)=(x, y)$ for $\ell=\overline{x, y}$ with $x, y \in X$.

The morphism $g$ is generically injective, as the points of contact $x, y$ of a bitangent $\ell$ clearly determine $\ell$ when $x \neq y$.

Lemma 8.3. The correspondence $\left[\Gamma_{X}\right]_{*}=6 \cdot \gamma: \mathrm{CH}^{2}(X) \rightarrow \mathrm{CH}^{2}(X)$ coincides with

$$
p_{*} \circ i^{*} \circ p^{*}: \mathrm{CH}^{2}(X) \rightarrow \mathrm{CH}^{2}\left(B_{X}\right) \rightarrow \mathrm{CH}^{2}\left(B_{X}\right) \rightarrow \mathrm{CH}^{2}(X) .
$$

The same assertion of course holds on the level of cohomology.

Mapping a bitangent $\ell \in F_{X}$ to its intersection $X \cap \ell$ defines a closed embedding

$$
F_{X} \hookrightarrow \operatorname{Hilb}^{2}(X) \text {. }
$$

The image is the fixed point locus of the Beauville involution mapping $[Z] \in \operatorname{Hilb}^{2}(X)$ to the residual intersection of the unique line through $Z$ with $X$.

Corollary 8.4. The surface $F_{X} \subset \operatorname{Hilb}^{2}(X)$ is a rigid Lagrangian surface of general type.

Proof. That $F_{X}$ is Lagrangian is an immediate consequence of $\gamma$ acting as - id on $H^{2,0}(X)$. It is rigid, because $H^{0}\left(F_{X}, \mathcal{N}_{F_{X} / \operatorname{Hilb}^{2}(X)}\right) \simeq H^{0}\left(F_{X}, \Omega_{X}\right)=0$ due to [Wel81, (3.43)]. Its canonical bundle $\omega_{F_{X}}$ is actually ample, as was shown in [Tik80, Wel81].

Remark 8.5. Using the geometric description of the bitangent correspondence, one can give another, more roundabout, proof of Corollary 8.2. Suppose $\gamma^{*} \neq-\mathrm{id}$ on $H^{2,0}(X)$. Then $q_{*} \circ p^{*}$ : $H^{2,0}(X) \longrightarrow H^{2,0}\left(F_{X}\right)$ does not vanish. By the work of Tikhomirov [Tik80] and Welters [Wel81], there exists an étale cover $\pi: F \rightarrow F_{X}$ of degree two with the property that $\bigwedge^{2} H^{1}(F, \mathbb{Q}) \simeq$ 


\section{CuRves AND CYCLES ON K3 SURFACES}

$\pi^{*} H^{2}\left(F_{X}, \mathbb{Q}\right)$. Here, $F$ is the Fano variety of lines on the double quartic solid $Y \rightarrow \mathbb{P}^{3}$ branched over $X \subset \mathbb{P}^{3}$. This would establish a nontrivial algebraic(!) correspondence between $H^{2}(X, \mathbb{Q})$ and $H^{1}(F, \mathbb{Q}) \otimes H^{1}(F, \mathbb{Q})$, that is, between the $\mathrm{K} 3$ surface $X$ and the square of the abelian variety given by the weight one Hodge structure of $F$. This is reminiscent of the Kuga-Satake correspondence which is conjectured to be algebraic. However, as François Charles explained to me, the ten-dimensional abelian variety determined by $H^{1}(F, \mathbb{Q})$ is too small to play a part in the Kuga-Satake correspondence for the generic quartic (which would be of the form $A^{2^{10}}$ with $A$ a simple abelian variety of dimension $2^{9}$ ), so that the uniqueness of the Kuga-Satake correspondence eventually leads to a contradiction. Hence, $\gamma^{*}=-$ id on $H^{2,0}(X)$.

Remark 8.6. Once Corollary 8.2 has been verified, it can in turn be used to prove Proposition 8.1 by considering the family of all quartics $\mathcal{X} \rightarrow|\mathcal{O}(4)|$. One uses either the explicit description for the Chow ring of $\mathrm{CH}^{*}(\mathcal{X})$ by viewing $\mathcal{X}$ as a projective bundle over $\mathbb{P}^{3}$ or a technique developed in [Voi12a] which only uses that $\mathcal{X}$ is rationally connected.

8.3. Consider now the curve of hyperflexes $D_{\mathrm{hf}} \subset F_{X}$ and the image of the curve $q^{-1}\left(D_{\mathrm{hf}}\right) \subset B_{X}$ under the projection $p: B_{X} \rightarrow X$. This yields the curve

$$
C_{\mathrm{hf}}:=p\left(q^{-1}\left(D_{\mathrm{hf}}\right)\right) \subset X
$$

of all points $x \in X$ such that there exists a hyperflex at $x$.

Proposition 8.7. For a quartic $X \subset \mathbb{P}^{3}$ not containing a line, the curve $C_{\mathrm{hf}} \subset X$ of contact points of hyperflexes is a constant cycle curve of order $n \mid 4$.

Proof. We shall first give two pointwise arguments showing that $C_{\mathrm{hf}}$ is a constant cycle curve. The first one seems to (wrongly?) suggest that the order should be at most two, whereas the second one can be turned into a rigorous argument proving the assertion.

- Any hyperflex $\ell$ at a point $x \in C_{\mathrm{hf}}$ is the limit of proper bitangents

$$
\ell_{t}:=\overline{x_{t}, y_{t}} \rightarrow \ell
$$

with $x_{t}, y_{t}$ both specializing to $x$. Since $\gamma\left(\left[x_{t}\right]\right)=\left[y_{t}\right]$, Proposition 8.1 shows that $\left[x_{t}\right]+\left[y_{t}\right]=2 \cdot c_{X}$, which after specializing gives $2 \cdot[x]=2 \cdot c_{X}$. Therefore, $C_{\mathrm{hf}}$ is a pointwise constant cycle curve and hence, by Proposition 3.7, also a constant cycle curve. Note that the argument cannot be used to actually prove that the order is $n \mid 4$ (or even better $n \mid 2$ ), as only $6 \cdot \gamma$ is a priori defined by an integral cycle and running the argument again with $6 \cdot \gamma$ only shows that the order divides 12 .

- For $x \in C_{\mathrm{hf}}$ there exists a line $\ell \subset T_{x} X$ with $\ell$ and $C_{x}$ intersecting only in $x$ (with multiplicity four). But $\ell=T_{x} X \cap H$ for some hyperplane $H \subset \mathbb{P}^{3}$. Hence, $4 \cdot[x]=C_{x} \cdot\left(\left.H\right|_{X}\right)=4 \cdot c_{X}$ by $(2.1)$.

- The last argument works for the base change $X_{k}$ to any field $k / \mathbb{C}$ (not necessarily algebraically closed), as long as the hyperflex $\ell$ is unique. Indeed, $T_{x} X_{k}$ and $C_{x} \subset X_{k}$ are defined over $k$ and if $\ell \subset T_{x} X_{\bar{k}}$ is the unique hyperflex, it is left invariant under $\operatorname{Gal}(\bar{k} / k)$. Hence, $\ell$ is and $H$ can be defined over $k$. Thus, $4 \cdot[x]=4 \cdot c_{X_{k}}$ in $\mathrm{CH}^{2}\left(X_{k}\right)$. This can be applied to the generic point $\eta$ of $C_{\mathrm{hf}} \subset X$ viewed as a closed point $\eta \in X \times k(\eta)$. Since $q^{-1}(D) \rightarrow C_{\mathrm{hf}}$ is generically injective (see the proof of Proposition 8.8), the generic point of $C_{\mathrm{hf}}$ admits a unique hyperflex. This proves that $\kappa_{C_{\mathrm{hf}}}$ is of order $n \mid 4$. 


\section{Huybrechts}

Continuing the analogy between the covering involutions of $X \rightarrow \mathbb{P}^{2}$ and $B_{X} \rightarrow F_{X}$ in the degree two and four case, respectively, the curve $C_{\mathrm{hf}} \subset X$ should be seen as the analogue of the degree six branching curve $C \subset X \rightarrow \mathbb{P}^{2}$. It is curious to note that as in the degree two case, the generic K3 surface of degree four contains a distinguished curve. It would be interesting to find distinguished curves in K3 surfaces of higher degree curve as well. ${ }^{5}$

8.4. A little more can be said about the curve $C_{\mathrm{hf}} \subset X$, which shall be recorded here.

Proposition 8.8. For a quartic $X \subset \mathbb{P}^{3}$ not containing a line the curve $C_{\mathrm{hf}} \subset X$ is a singular irreducible curve in the linear system $\left|\mathcal{O}_{X}(20)\right|$ of geometric genus 201. In particular, $C_{\mathrm{hf}}$ is not rational.

Proof. By construction, $C_{\mathrm{hf}}$ is the image of $q^{-1}\left(D_{\mathrm{hf}}\right) \simeq D_{\mathrm{hf}} \subset F_{X}$, where $q: B_{X} \rightarrow F_{X}$ is a $2: 1$ cover with ramification curve $D_{\mathrm{hf}} \subset F_{X}$. By [Wel81, (1.6)\& p.40] the curve of hyperflexes $D_{\mathrm{hf}} \subset F_{X}$ is a smooth curve of genus 201.

A dimension count similar to the one in [Wel81, p. 17-18] shows that $q^{-1}\left(D_{\mathrm{hf}}\right) \rightarrow C_{\mathrm{hf}}$ is generically injective. Also, using the notation of [Wel81, Sec. 3], one computes the intersection number $p^{*} h \cdot\left[q^{-1}\left(D_{\mathrm{hf}}\right)\right]=p^{*} h \cdot\left(\sigma+p^{*} h\right)=80$, where $h$ is the hyperplane section of $X$ and $\sigma$ is the hyperplane section on the space of lines. Since $h . h=4$, this shows that $\left[C_{\mathrm{hf}}\right]=20 \cdot h$, that is, $C_{\mathrm{hf}} \in\left|\mathcal{O}_{X}(20)\right|$.

Remark 8.9. Let us go back to the variety of bitangents $F_{X} \subset \operatorname{Hilb}^{2}(X)$. As pointed out by Kieran O'Grady, $\mathrm{Hilb}^{2}(X)$ can be seen as a degeneration of an EPW-sextic, so that $F_{X}$ corresponds to the singular locus of the sextic. In the same spirit, whenever an EPW-sextic is of the form $\operatorname{Hilb}^{2}\left(X^{\prime}\right)$ for some K3 surface $X^{\prime}$, the singular locus of the sextic is likely to give an interesting antisymplectic auto-correspondence for $X^{\prime}$. This can be applied to a generic K3 surface of genus six, see [OG10, Sec. 4]. This should eventually lead to a picture quite similar to the one described here. In particular, one finds as an analogue of the curve $C_{\mathrm{hf}}$ the curve of points in $X^{\prime}$ with a conic in a certain ambient Fano threefold with higher contact at this point which is expected to be a constant cycle curve.

Remark 8.10. We conclude by making two remarks on the dynamical aspects of the bitangent correspondence.

i) One may wonder whether the bitangent correspondence $x \longmapsto y_{i}$ can play the role of an endomorphism in other respects as well. For example, endomorphisms of K3 surfaces have been used to prove potential density of rational points on K3 surfaces over number fields. Although the universal family of bitangents is also defined over the same field as $X$, the construction seems of little use for this purpose. Indeed, the surface $B_{X}$ is of general type and Lang's conjecture would predict much fewer rational points on $B_{X}$ than on $X$ itself. In other words, mapping $x \in X$ to the other points of contact $y_{i} \in X$ of bitangents at $x$ increases the residue field.

ii) If $C \subset X$ is a constant cycle curve in an arbitrary K3 surface and $f: X \stackrel{\sim}{\longrightarrow} X$ is any automorphism, then $f(C) \subset X$ is again a constant cycle curve (of the same order). But, since the generic K3 surface does not admit nontrivial automorphisms, this method fails in the generic case. However, for the generic quartic $X \subset \mathbb{P}^{3}$ the bitangent correspondence can sometimes replace the missing automorphisms. Indeed, if $C \subset X$ is a constant cycle curve, then $p\left(i\left(p^{-1}(C)\right)\right)$

\footnotetext{
${ }^{5}$ To venture a guess, any distinguished curve, that is, a curve that is naturally defined in all generic K3 surfaces of fixed degree, should be a constant cycle curve. Compare this to O'Grady's Franchetta conjecture in [OG13].
} 


\section{Curves And CYCles on K3 SuRfaCes}

is again a constant cycle curve. This can be applied to for example $C=C_{\mathrm{hf}}$, in which case $p^{-1}(C)=q^{-1}\left(D_{\mathrm{hf}}\right) \cup C^{\prime}$. The curve $q^{-1}\left(D_{\mathrm{hf}}\right)$ is of course invariant under the involution $i$, but $C^{\prime}$ is not and produces a new constant cycle curve $p\left(i\left(C^{\prime}\right)\right)$ in $X$.

\section{Finite fields}

In this section we study K3 surfaces over finite fields $\mathbb{F}_{q}, q=p^{d}$ with $p \neq 2$. Contrary to the case of $\mathrm{K} 3$ surfaces over $\overline{\mathbb{Q}}$, it is easy to see that $\mathrm{CH}^{2}(X) \simeq \mathbb{Z}$ for any $X$ over $\overline{\mathbb{F}}_{p}$. Indeed, any finite collection of points is contained in some curve $C \subset X$. As one always finds a finite field $\mathbb{F}_{q} \subset \overline{\mathbb{F}}_{p}$ over which the finitely many points and the curve are defined, the finiteness of $\mathbb{F}_{q}$-rational points of $\operatorname{Pic}^{0}(C)$ and the torsion freeness of $\mathrm{CH}^{2}(X)$ (over the algebraically closed field $\overline{\mathbb{F}}_{p}$ ) are enough to conclude.

But the Bloch-Beilinson philosophy in positive characteristic also predicts $\mathrm{CH}^{2}(X) \simeq \mathbb{Z}$ for $X$ over the algebraic closure of $\mathbb{F}_{p}(t)$. Surprisingly, explicit examples of K3 surfaces over function fields verifying the conjecture can actually be worked out, unlike the case of K3 surfaces over number fields.

Remark 9.1. For $k=\overline{\mathbb{F}}_{p}$ the Chow group is not expected to increase when passing from $X$ over $k$ to $X \times_{k} \overline{k(t)}$. This does not hold for other fields. Indeed, similarly to Bloch's argument showing that $\mathrm{CH}^{2}(X) \rightarrow \mathrm{CH}^{2}\left(X \times_{k} k\left(\eta_{X}\right)\right)$ is not surjective for a $\mathrm{K} 3$ surface $X$ in arbitrary characteristic as long as $\rho(X) \neq 22$, Green, Griffiths, and Paranjape showed in [GGP04] that in characteristic zero the Chow group already grows when passing to an algebraically closed field of transcendence degree one over the base field. See Corollary 3.11 for a weaker version that also works in positive characteristic.

9.1. Due to the isomorphism $\mathrm{CH}^{2}(X) \simeq \mathbb{Z}$ for a $\mathrm{K} 3$ surface $X$ over $\overline{\mathbb{F}}_{p}$, any curve $C \subset X$ is a pointwise constant cycle curve. But the stronger statement is also conjectured to hold due to the following.

Proposition 9.2. Let $X$ be a $K 3$ surface over $\overline{\mathbb{F}}_{p}$. Then $X \times_{\overline{\mathbb{F}}_{p}} \overline{\mathbb{F}_{p}(t)}$ satisfies the Bloch-Beilinson conjecture, that is, $\mathrm{CH}^{2}\left(X \times_{\overline{\mathbb{F}}_{p}} \overline{\mathbb{F}_{p}(t)}\right) \simeq \mathbb{Z}$, if and only if every curve $C \subset X$ is a constant cycle curve.

Proof. Clearly, $\mathrm{CH}^{2}\left(X \times_{\overline{\mathbb{F}}_{p}} \overline{\mathbb{F}_{p}(t)}\right) \simeq \mathbb{Z}$ if and only if the pull-back

$$
\mathrm{CH}^{2}(X) \rightarrow \mathrm{CH}^{2}\left(X \times_{\overline{\mathbb{F}}_{p}} \overline{\mathbb{F}_{p}(t)}\right)
$$

is an isomorphism. Due to Lemma 3.8, a curve $C \subset X$ is a constant cycle curve if and only if $\left[\eta_{C}\right] \in \mathrm{CH}^{2}\left(X \times \overline{k\left(\eta_{C}\right)}\right)$ is in the image of the pull-back. Assuming the Bloch-Beilinson conjecture, the latter now follows from choosing an embedding $\overline{\mathbb{F}}_{p} \subset k\left(\eta_{C}\right) \subset \overline{k\left(\eta_{C}\right)} \simeq \overline{\mathbb{F}_{p}(t)}$.

Conversely, a closed point $x \in X \times \overline{\mathbb{F}_{p}(t)}$ projects to either a closed point in $X$ or the generic point of a curve $C \subset X$. In the first case, $[x]$ is in the image of $(9.1)$, whereas in the latter $[x]=\left[\eta_{C}\right]$ with the generic point $\eta_{C} \in C$ viewed as a closed point of $X \times \overline{k\left(\eta_{C}\right)}=X \times \overline{\mathbb{F}_{p}(t)}$. But if $C$ is a constant cycle curve, then up to torsion $\left[\eta_{C}\right]$ is in the image of $\mathrm{CH}^{2}(X) \rightarrow \mathrm{CH}^{2}\left(X \times k\left(\eta_{C}\right)\right)$ and, therefore, $[x]$ is in the image of $(9.1)$.

We stress again that due to [GGP04], the corresponding statement is false for $X$ over $\overline{\mathbb{Q}}$, that is, there always exist curves $C \subset X$ which are not constant cycle curves. However, according to 


\section{HuYBREchts}

Conjecture, 2 all curves are expected to be pointwise constant cycle curves and it seems likely that every point is at least contained in a constant cycle curve (cf. Conjecture 4). Note that any linear system $|L|$ could contain countably many constant cycle curves. So a priori the finiteness of constant cycle curves of bounded order does not prove the existence of curves that are not constant cycle curves. The existence for surfaces over $\overline{\mathbb{Q}}$ as proved in [GGP04] eventually relies on results of Terasoma and in fact shows the existence of infinitely many such curves in any ample linear system.

9.2. The Bloch-Beilinson conjecture for function fields is related to the conjectured finite-dimensionality in the sense of Kimura and O'Sullivan. To be more precise, consider a K3 surface $X$ and a curve $C \subset X$ over a finite field $\mathbb{F}_{q}$. Suppose Kimura-O'Sullivan finite-dimensionality holds for $X$ and $\widetilde{C}$ and hence for $X \times \widetilde{C}$ (cf. [Kim05, Prop. 5.10]). Then the Tate conjecture $T^{1}$ for $X$ (using the recent [Cha12, Mad13, Mau12]) and $\widetilde{C}$, which implies the Tate conjecture $T^{1}$ for $X \times \widetilde{C}$ and by duality the Tate conjecture $T^{2}$ for $X \times \widetilde{C}$ (see [Tat94] or [Mil07, Cor. 2.2, Thm. 1.4]), yields

$$
\mathrm{CH}^{2}(X \times \widetilde{C}) \otimes_{\mathbb{Z}} \mathbb{Q}_{\ell} \stackrel{\sim}{\longrightarrow} H^{4}\left((X \times \widetilde{C})_{\overline{\mathbb{F}}_{q}}, \mathbb{Q}_{\ell}(2)\right)^{\operatorname{Gal}\left(\overline{\mathbb{F}}_{q} / \mathbb{F}_{q}\right)}
$$

see [Jan07, Thm. 0.4, Thm. 5.2], [Ka03, Thm. 1], or [And05, Thm. 4.2]. The cycle $Z_{C}$ is homologically trivial, that is,

$$
0=\left[Z_{C}=\Delta_{f_{C}}-C \times\left[L_{0}\right]-\left\{x_{0}\right\} \times \widetilde{C}\right] \in H^{4}\left((X \times \widetilde{C})_{\overline{\mathbb{F}}_{q}}, \mathbb{Q}_{\ell}(2)\right)^{\operatorname{Gal}\left(\overline{\mathbb{F}}_{q} / \mathbb{F}_{q}\right)}
$$

(see Section 5.1) and, under the assumption of Kimura-O'Sullivan finite-dimensionality, the cycle $Z_{C}$ on $X \times \widetilde{C}$ must then also be rationally equivalent to zero up to torsion. Hence, its restriction $\kappa_{C} \in \mathrm{CH}^{2}\left(X \times k\left(\eta_{C}\right)\right)$ is also torsion. Thus, the Bloch-Beilinson conjecture holds for the K3 surface $X \times \mathbb{F}_{q}(t)$, that is, $\mathrm{CH}^{2}\left(X \times \overline{\mathbb{F}_{q}(t)}\right) \simeq \mathbb{Z}$.

This remark immediately produces actual examples of K3 surfaces over function fields for which the Bloch-Beilinson conjecture can be confirmed. Note that this is in contrast to the number field case where not a single $\mathrm{K} 3$ surface is known to satisfy $\mathrm{CH}^{2}\left(X_{\overline{\mathbb{Q}}}\right) \simeq \mathbb{Z}$. It would also be very interesting to find an example of a nonisotrivial K3 surface over $\overline{\mathbb{F}_{p}(t)}$ satisfying the Bloch-Beilinson conjecture.

Example 9.3. Kimura-O'Sullivan finite-dimensionality is known for rational Chow motives in the tensor subcategory generated by Chow motives of abelian varieties, cf. [Kim05, Ex. 9.1]. Also, quotients of finite-dimensional Chow motives are again finite dimensional. Hence, Kummer surfaces are known to be finite-dimensional. Therefore, any Kummer surface $X$ over $\mathbb{F}_{q}$ yields a K3 surface $X_{\mathbb{F}_{q}(t)}$ over $\mathbb{F}_{q}(t)$ which satisfies the Bloch-Beilinson conjecture, that is, $\mathrm{CH}^{2}\left(X \times \overline{\mathbb{F}_{q}(t)}\right) \simeq$ $\mathbb{Z}$. Finite dimensionality of Kummer surfaces is eventually deduced from the fact that they are dominated by products of curves and, in fact, in [Sch87] the Bloch-Beilinson conjecture for $X \times \mathbb{F}_{q}(t)$ was proved for any variety $X$ dominated by a product of curves.

The reduction modulo $p$ of Voisin's example in [Voi96, Sect. 3.3] is likely to provide other examples. See [Ped12] for more on finite-dimensionality of K3 surfaces (in characteristic zero).

Proposition 9.4. Let $X$ be a $K 3$ surface over a finite field $\mathbb{F}_{q}$ for which Kimura-O'Sullivan finite-dimensionality holds, for example $X$ a Kummer surface. Then every curve in $X_{\overline{\mathbb{F}}_{q}}$ is a constant cycle curve.

By Proposition 4.2, the result also holds true for unirational (and hence by [Lie13] for all supersingular) K3 surfaces. 


\section{Curves AND CyCles ON K3 SuRfaCes}

Remark 9.5. In the situation of the corollary and assuming that $X$ is not unirational, it would be interesting to decide whether among the curves in a fixed linear system $|L|$, which are all constant cycle curves, there are at most finitely many of bounded order, that is, whether the analogue of Proposition 5.1 holds. Also, is there an explicit example (for which Kimura-O'Sullivan finitedimensionality is known) where one can show that all curves are constant cycle curves directly (i.e. geometrically)?

9.3. We conclude this section with a result lending evidence to Conjecture 4 .

Proposition 9.6. Every closed point $x \in X$ in a K3 surface over $\overline{\mathbb{F}}_{p}$ is contained in a constant cycle curve $x \in C \subset X$.

Proof. If $X$ is unirational, then every point $x \in X$ is contained in a rational curve, which yields the assertion. Thus, we may assume that $X$ is not unirational.

Next we use the construction of Section 6.2. So we pick a smooth elliptic surface $\mathcal{C} \rightarrow T$ with a surjective morphism $p: \mathcal{C} \longrightarrow X$. As before, by a further base change, we can assume that $\mathcal{C} \rightarrow T$ comes with a zero-section that maps to a constant cycle curve in $X$. Now, all singular fibres are rational and, therefore, map to constant cycle curves in $X$. A point $x \in \mathcal{C}_{t}$ in a smooth fibre is automatically torsion and hence contained in one of the curves $C_{n} \subset \mathcal{C}$ of fibrewise torsion points. By Section 6.2, the curves $C_{n}$ are constant cycle curves. This proves the assertion.

Note that a stronger result for Kummer surfaces, namely the existence of a rational(!) curve through every point, has been proved already by Bogomolov and Tschinkel in [BT05]. The assumption that $X$ is Kummer is used in [BT05] for an explicit geometric construction for Jacobian Kummer surfaces.

\section{Further questions and remarks}

10.1. Let $X$ be a K3 surface over an arbitrary algebraically closed field $k$. It seems not impossible that any closed point $x \in X$ with $[x]=c_{X}$ is in fact contained in a constant cycle curve $C \subset X$ (see Conjecture 4). Corollary 3.10 provides some weak evidence. Note however that this should fail in general (probably whenever $k$ is uncountable and algebraically closed) when constant cycle curves are replaced by rational curves. For example, for a complex K3 surface and a nonrational constant cycle curve $C \subset X$ (examples have been given above), only countably many of the points $x \in C$ can be contained in some rational curve. Indeed, there are at most countably many rational curves contained in $X$.

10.2. By Proposition 5.1, on a complex K3 surface only finitely many curves in a fixed linear system $|L|$ can be constant cycle curves of a given order $n$. This prompts two questions:

(i) Can the number be determined, for example in geometrically interesting situations?

(ii) Is this number deformation invariant?

Both aspects have been addressed extensively for rational curves in the context of the YauZaslow conjecture, see [KMPS10]. Once the counting is done properly, the number of rational curves (counted with multiplicities) is deformation invariant and is given by a certain generating series.

For the time being it is not at all clear (to me) whether a similar picture is to be expected for constant cycle curves. In fact, the observation that in addition to from rational curves, 


\section{Huybrechts}

nonrational curves (even smooth ones) can also occur as constant cycle curves of order one is a little suspicious. One would need to add those to the (Gromov-Witten) number of rational curves.

10.3. It is to be expected that an analogous picture will emerge for curves $C \subset X$ that are special with respect to O'Grady's filtration (see [OG13]):

$$
S_{0}(X) \subset S_{1}(X) \subset \ldots \subset S_{d}(X) \subset \ldots \subset \mathrm{CH}^{2}(X) .
$$

Here, $S_{d}(X)$ is the set of cycles that can be written as $[Z]+m \cdot c_{X}$ with $Z$ an effective cycle of degree $d$. Equivalently, $\alpha \in S_{d}(X)$ if and only if there exists a possibly reducible curve $C \subset X$ such that $g(\widetilde{C}) \leqslant d$ and $\alpha \in \operatorname{Im}\left(f_{C *}: \operatorname{Pic}(\widetilde{C}) \rightarrow \mathrm{CH}^{2}(X)\right)$ (see [OG13, Voi12b]). In particular, $S_{0}(X)=\mathbb{Z} \cdot c_{X}$.

Note that the kernel of $f_{C *}: \operatorname{Pic}^{0}(\widetilde{C}) \rightarrow \mathrm{CH}^{2}(X)$ is a countable union of translates $L_{i}+A_{i}$ of abelian subvarieties $A_{i} \subset \operatorname{Pic}^{0}(\widetilde{C})$. One defines $\operatorname{dim}\left(\operatorname{Ker}\left(f_{C *}\right)\right)$ as the minimum of all dimensions $\operatorname{dim}\left(A_{i}\right)$. So, $\operatorname{dim}\left(\operatorname{Ker}\left(f_{C *}\right)\right)=g$ if and only if $C$ is a constant cycle curve (we work over $\mathbb{C}$ ), which is equivalent to $\operatorname{Im}\left(f_{C *}\right) \subset S_{0}(X)$. More generally, it is not difficult to see that $\operatorname{dim}\left(\operatorname{Ker}\left(f_{C *}\right)\right) \geqslant$ $g-d$ implies $\operatorname{Im}\left(f_{C *}\right) \subset S_{d}(X)$ and I would expect that with the techniques from [Voi12c] the converse can also be proved.

Then in any linear system $|L|$, the locus of curves $C$ with $\operatorname{Im}\left(f_{C *}\right) \subset S_{d}(X)$ should be a countable union of Zariski closed subsets, but in order to get honest Zariski closed subsets, one would first need to introduce the analogue of the order of a constant cycle curve and then restrict to those of finite order.

For a lack of a better name one could call a curve $C \subset X$, say integral, $d$-special if $\operatorname{Im}\left(f_{C *}\right) \subset$ $S_{d}(X)$. Of course, there would not be anything special about $C$ for $d \geqslant g(\widetilde{C})$ and 0 -special curves would simply be constant cycle curves. So the first question one needs to address is how to define the order of a $d$-special curve for $d<g(\widetilde{C})$.

10.4. We have not yet explored constant cycle curves from the infinitesimal point of view. As explained by Bloch, $H^{2}\left(X, \mathcal{O}_{X}\right) \otimes \Omega_{\mathbb{C} / \mathbb{Q}}$ should be viewed as the 'tangent space' of $\mathrm{CH}^{2}(X)$. For any curve $C \subset X$, the induced map $f_{C *}: \operatorname{Pic}^{0}(\widetilde{C}) \rightarrow \mathrm{CH}^{2}(X)$ induces a natural map between the tangent spaces $H^{1}\left(\widetilde{C}, \mathcal{O}_{\widetilde{C}}\right) \rightarrow H^{2}\left(X, \mathcal{O}_{X}\right) \otimes \Omega_{\mathbb{C} / \mathbb{Q}}$. For a constant cycle curve the map should be trivial and, as addressed in [GG05], it is interesting to see how much information about $f_{C *}$ is actually encoded by the derivatives (at all points). Is there anything special about the derivative in points $x \in C$ with $[x]=c_{X}$ ?

\section{Appendix}

\section{Claire Voisin}

The aim of this appendix is to prove Conjecture 3 for the generic (and not only general!) K3 surface. More precisely, we prove the following result.

Theorem A.1. Let $X$ be a complex K3 surface admitting a nonisotrivial one-parameter family of elliptic curves which is not an elliptic pencil. Then $X$ admits infinitely many constant cycle curves of bounded order, the union of which is dense in the classical topology. 


\section{Curves AND CYCles on K3 SuRfaces}

Since the assumptions are Zariski open conditions and families of elliptic curves satisfying the assumptions can be found for the general K3 surface of Picard number one, the theorem does imply Conjecture 3 for the generic complex K3 surface.

A.1. To set up notation, we spell out the assumptions of the theorem: We assume that there exist a smooth elliptic surface $q: \mathcal{C} \longrightarrow T$ and a surjective morphism $p: \mathcal{C} \rightarrow X$ with the following properties:

i) The family $q: \mathcal{C} \longrightarrow T$ is not isotrivial, that is, there does not exist any dominant quasi-finite morphism $T^{\prime} \longrightarrow T$ such that the base change $q^{\prime}: \mathcal{C}^{\prime}:=\mathcal{C} \times_{T} T^{\prime} \rightarrow T^{\prime}$ is a trivial family of elliptic curves.

ii) For generic $y \in X$ there exist at least two smooth fibres $\mathcal{C}_{t}, \mathcal{C}_{t^{\prime}} \subset \mathcal{C}$ such that $p\left(\mathcal{C}_{t}\right)$ and $p\left(\mathcal{C}_{t^{\prime}}\right)$ intersect transversally in $y$.

As in Section 6.2, we can always reduce to the situation that $q: \mathcal{C} \rightarrow T$ comes with a zerosection, the image of which is a constant cycle curve in $X$ (we could even assume the image to be a rational curve). The origin of a smooth fibre $\mathcal{C}_{t}$ fixed by the zero section will be called $x_{t}$ and torsion in the fibres is considered with respect to this choice.

A.2. The technique to produce new constant cycle curves is to multiply a given one with respect to the additive structure of the fibres of $q: \mathcal{C} \rightarrow T$. Under the above hypotheses one proves the following result.

Lemma A.2. Assume that $D \subset \mathcal{C}$ is a multi-section of $q: \mathcal{C} \rightarrow T$ such that

(i) the image $C:=p(D)$ is a constant cycle curve of order $n$;

(ii) the intersection of $D$ with the generic fibres $\mathcal{C}_{t}$ contains a nontorsion point.

Then there exists an $N>0$ such that the union of all constant cycle curves of order $\leqslant N$ is dense in the classical topology.

Proof. If $C \subset X$ is the image of a finite morphism $f: D \rightarrow X$ from an integral curve $D$, then the image $\kappa_{C} \times k\left(\eta_{D}\right)$ of $\kappa_{C}$ under the base change map

$$
\mathrm{CH}^{2}\left(X \times k\left(\eta_{C}\right)\right) \rightarrow \mathrm{CH}^{2}\left(X \times k\left(\eta_{D}\right)\right)
$$

can be described as the restriction of $\left[\Gamma_{f}-\left\{x_{0}\right\} \times D\right] \in \mathrm{CH}^{2}(X \times D)$.

Next, consider fibrewise multiplication by $m$ for the family $q: \mathcal{C} \longrightarrow T$, which defines a morphism $\mu_{m}: U \rightarrow U$ on some open set $U \subset \mathcal{C}$. Denote the graphs of the two morphisms $p: U \rightarrow X$ and $p_{m}:=p \circ \mu_{m}: U \rightarrow X$ by $\Gamma$ and $\Gamma_{m}$, respectively. We claim that, after shrinking $U$ if necessary, there exists an $N_{m}>0$ such that

$$
N_{m} \cdot\left(\left[\Gamma_{m}-\left\{x_{0}\right\} \times U\right]-m \cdot\left[\Gamma-\left\{x_{0}\right\} \times U\right]\right)=0 \text { in } \mathrm{CH}^{2}(X \times U) .
$$

Indeed, the correspondence $\left[\Gamma_{m}\right]_{*}: \mathrm{CH}^{2}(U) \rightarrow \mathrm{CH}^{2}(X)$ maps the class of a point $x \in \mathcal{C}_{t}$ to $m \cdot[x]+(1-m) \cdot\left[x_{t}\right]$, where $x_{t} \in \mathcal{C}_{t}$ is the origin of the elliptic curve $\mathcal{C}_{t}$. Since $\left[p\left(x_{t}\right)\right]=\left[x_{0}\right]$, one finds $\left[\Gamma_{m}-\left\{x_{0}\right\} \times U\right]_{*}=m \cdot\left[\Gamma-\left\{x_{0}\right\} \times U\right]_{*}: \mathrm{CH}^{2}(U) \rightarrow \mathrm{CH}^{2}(X)$. Using Bloch-Srinivas [BS83], one obtains the existence of $N_{m}$ (after shrinking $U$ if necessary).

Let now $D \subset \mathcal{C}$ be as assumed. Fix $m$ and choose $N_{m}$ above such that $n \mid N_{m}$. Then define $D\left(m^{k}\right):=\mu_{m^{k}}(D)=\mu_{m}^{k}(D)$ (or, more precisely, $\overline{\mu_{m^{k}}(D \cap U)}$ ) and $C\left(m^{k}\right):=p\left(D\left(m^{k}\right)\right)=$ $p_{m^{k}}(D)$. Since $\kappa_{C} \times k\left(\eta_{D}\right)$ is the specialization of $\left[\Gamma_{p}-\left\{x_{0}\right\} \times U\right]$ and similarly for all $C\left(m^{k}\right)$, 


\section{HuYbrechts}

one finds

$$
N_{m} \cdot\left(\kappa_{C\left(m^{k}\right)} \times k\left(\eta_{D}\right)-m \cdot \kappa_{C\left(m^{k-1}\right)} \times k\left(\eta_{D}\right)\right)=0
$$

in $\mathrm{CH}^{2}\left(X \times k\left(\eta_{D}\right)\right)$. Since $n \mid N_{m}$ and, therefore, $N_{m} \cdot \kappa_{C}=0$, one obtains by recursion that $N_{m} \cdot\left(\kappa_{C\left(m^{k}\right)} \times k\left(\eta_{D}\right)\right)=0$ for all $k$.

The base change induced by $p_{m^{k}}: D \longrightarrow C\left(m^{k}\right)$ factorizes via

$$
\mathrm{CH}^{2}\left(X \times k\left(\eta_{C\left(m^{k}\right)}\right)\right) \rightarrow \mathrm{CH}^{2}\left(X \times k\left(\eta_{D\left(m^{k}\right)}\right)\right) \rightarrow \mathrm{CH}^{2}\left(X \times k\left(\eta_{D}\right)\right) .
$$

Since $\operatorname{deg}\left(p: D\left(m^{k}\right) \longrightarrow C\left(m^{k}\right)\right) \leqslant \operatorname{deg}(p: \mathcal{C} \longrightarrow X)$ and $\operatorname{deg}\left(\mu_{m^{k}}: D \rightarrow D\left(m^{k}\right)\right) \leqslant\left(D \cdot \mathcal{C}_{t}\right)$, there exists an $N^{\prime}>0$ independent of $m$ and $k$, such that the kernel of (A.1) is annihilated by $N^{\prime}$. This shows that the curves $C\left(m^{k}\right)$ are constant cycle curves of order $\leqslant N_{m} \cdot N^{\prime}$.

If the intersection of $D$ with the generic fibre $\mathcal{C}_{t}$ contains a nontorsion point, then the union $\bigcup D\left(m^{k}\right) \subset \mathcal{C}$ of constant cycle curves of order $\leqslant N_{m} \cdot N^{\prime}$ is dense in the Zariski topology and so is its image $\bigcup C\left(m^{k}\right) \subset X$.

To obtain density in the classical topology one needs to prove that the integers $N_{m}$ can be chosen independent of $m$. Consider the map $\sigma: \mathcal{C} \times{ }_{T} \mathcal{C} \rightarrow \mathcal{C} \rightarrow X$ that is given as the composition of the summation in the fibres $\mathcal{C}_{t}$ with the projection $p$. For points $x, x^{\prime} \in \mathcal{C}$ in a smooth fibre $\mathcal{C}_{t}$, we have $\left[p\left(x+x^{\prime}\right)\right]=[p(x)]+\left[p\left(x^{\prime}\right)\right]-c_{X}$ in $\mathrm{CH}^{2}(X)$, as by assumption the class of the image $p\left(x_{t}\right)$ of the origin $x_{t} \in \mathcal{C}_{t}$ is $c_{X}$. Now consider the codimension two cycle

$$
\Gamma:=\Gamma_{\sigma}-\Gamma_{p \circ p r_{1}}-\Gamma_{p \circ p r_{2}}+\left\{x_{0}\right\} \times \mathcal{C} \times_{T} \mathcal{C}
$$

on $X \times \mathcal{C} \times_{T} \mathcal{C}$. Then by Bloch-Srinivas [BS83], there exists an integer $N$ such that $N \cdot[\Gamma]=0$ in $\mathrm{CH}^{2}(X \times V)$ for some nonempty open subset $V \subset \mathcal{C} \times_{T} \mathcal{C}$.

Restriction to the diagonal in $\mathcal{C} \times_{T} \mathcal{C}$ yields

$$
N \cdot\left(\left[\Gamma_{2}\right]-2 \cdot[\Gamma]+\left[\left\{x_{0}\right\} \times W\right]\right)=0
$$

in $\mathrm{CH}^{2}(X \times W)$ for a certain non-empty open set $W \subset \mathcal{C}$ (the restriction of $V$ with the diagonal). By induction on $m$ and by restricting the cycle $\Gamma$ to the surface that is given as the image of id $\times \mu_{m-1}: \mathcal{C} \longrightarrow \mathcal{C} \times_{T} \mathcal{C}, x \longmapsto(x,(m-1) \cdot x)$, one proves similarly that

$$
N \cdot\left(\left[\Gamma_{m}\right]-m \cdot[\Gamma]+(m-1) \cdot\left[\left\{x_{0}\right\} \times W\right]\right)=0
$$

in $\mathrm{CH}^{2}(X \times W)$.

As before, this shows that all curves $C(m)$ are constant cycle curves of order $\leqslant N \cdot N^{\prime}$ (independent of $m$ ). If the generic fibre $\mathcal{C}_{t}$ is viewed as a torus $\mathbb{R}^{2} / \mathbb{Z}^{2}$, then the intersection of $\bigcup D(m)$ with $\mathcal{C}_{t}$ contains a set of the form $\mathbb{Z} \cdot\left(x_{1}, x_{2}\right) \subset \mathbb{R}^{2} / \mathbb{Z}^{2}$ with $x_{1}$ or $x_{2}$ nontorsion. Therefore, the closure of $\mathcal{C}_{t} \cap \bigcup D(m)$ is either the full torus $\mathcal{C}_{t}=\mathbb{R}^{2} / \mathbb{Z}^{2}$ or a finite union of translates of circles $\mathbb{S}^{1} \subset \mathcal{C}_{t}=\mathbb{R}^{2} / \mathbb{Z}^{2}$.

The second possibility is ruled out, since the monodromy action on $H_{1}\left(\mathcal{C}_{t}, \mathbb{Z}\right)$ would then act via a finite group on the class of the finite union of circles, contradicting the fact that the monodromy group is of finite index in $\mathrm{Sl}(2, \mathbb{Z})$. The argument can be made more explicit as follows. If the intersection is generically not dense, then the components of its closure satisfy a linear equation of the form $a \cdot x_{1}+b \cdot x_{2}=0$ with $a, b \in \mathbb{Z}$ not both zero. If assumed coprime, $a$ and $b$ are unique up to sign. Such an equation exists for a countable union of real analytic subsets of $D$ over the regular locus of $q$. Thus, $(a, b)$ is locally constant up to sign and defines a section of the pull-back of $R^{1} q_{*} \mathbb{Z}$ to a dense open subset of $D$ (or rather the appropriate double cover that accommodates for the sign ambiguity). The latter contradicts the nonisotriviality of $\mathcal{C} \times_{T} D \rightarrow D$. 


\section{Curves AND CYCles on K3 SuRfaces}

The last part of the proof is inspired by a similar argument due to Chen and Lewis in [CL13].

A.3. Let $\mathcal{C}_{n} \subset \mathcal{C}$ be the closure of the set of all $n$-torsion points $x \in \mathcal{C}_{t}$ in smooth fibres $\mathcal{C}_{t}$ and let $C_{n}:=p\left(\mathcal{C}_{n}\right)$. Then by Section 6.2 the curve $C_{n}$ is a (possibly reducible) constant cycle curve of order $d \mid n$. Of course, the curves $\mathcal{C}_{n}$ cannot be used as input for Lemma A.2, but the curve $\widetilde{\mathcal{C}}_{n}:=p^{-1}\left(p\left(\mathcal{C}_{n}\right)\right)$ might contain another component $D$ satisfying assumptions (i) (which is automatic) and (ii) of Lemma A.2. We will argue by contradiction and assume that

(*) if $D \subset p^{-1}\left(p\left(\mathcal{C}_{n}\right)\right)=p^{-1}\left(C_{n}\right)$ is an irreducible component, then its intersection with the generic fibres $\mathcal{C}_{t}$ contains only torsion points.

Consider a multi-section $D \subset \mathcal{C}$. We call $D$ flat at a point $x \in D$ if locally analytically $D$ can be lifted to a flat section of $H_{\mathbb{C}}^{1}:=R^{1} q_{*} \mathbb{C}$. For example, the multi-sections $\mathcal{C}_{n}$ are flat in all points of intersection with smooth fibres. Note that the notion makes sense for locally analytic sections as well.

Lemma A.3. Suppose $D \subset \mathcal{C}$ is an irreducible multi-section of $q: \mathcal{C} \rightarrow T$ and assume it is flat locally around a point $x \in D$ which is a nontorsion point in a smooth fibre $\mathcal{C}_{t}$. Then $q: \mathcal{C} \rightarrow T$ is isotrivial (contradicting assumption (i) in A.1).

Proof. The assumption immediately implies that $D$ is flat in all points of intersection with smooth fibres. Consider the base change $q_{D}: \mathcal{C} \times_{T} D \rightarrow D$. In addition to the pull-back of the zero-section, it comes with a natural flat section which is fibrewise nontorsion. This flat section of $q_{D}$ defines a section of $R^{1} q_{D *} \mathbb{C} / R^{1} q_{D *} \mathbb{Z}$ (over the regular part) and, therefore, corresponds to a monodromy invariant element of $\mathbb{C}^{2} / \mathbb{Q}^{2}$. But for a nonisotrivial family the monodromy group is a finite index subgroup $\Gamma \subset \operatorname{Sl}(2, \mathbb{Z})$ and thus $\left(\mathbb{C}^{2} / \mathbb{Q}^{2}\right)^{\Gamma}=0$.

Let now $x, x^{\prime} \in \mathcal{C}$ be generic points with $p(x)=p\left(x^{\prime}\right)$ and $x \in D \subset \mathcal{C}$ a local multi-section through $x$. Consider a component $D^{\prime} \subset p^{-1}(p(D))$ through $x^{\prime}$. A priori, $D$ might be flat at $x$ without $D^{\prime}$ being flat at $x^{\prime}$. However, if $x$ is $n$-torsion in the fibre $\mathcal{C}_{t}, t=q(x)$, then $x \in \mathcal{C}_{n}$ and hence $x^{\prime} \in \widetilde{\mathcal{C}}_{n}$. So assuming $(*)$, the components of $\widetilde{\mathcal{C}}_{n}$ containing $x^{\prime}$ are again contained in some $\mathcal{C}_{m}$ and are, therefore, flat multi-sections of $q: \mathcal{C} \longrightarrow T$. Using a density argument, this is enough to conclude the general case.

Lemma A.4. Let $x, x^{\prime} \in \mathcal{C}$ be generic points with $p(x)=p\left(x^{\prime}\right)$. Assume that $x \in D \subset \mathcal{C}$ is a local analytic section through $x$ and that $D^{\prime} \subset p^{-1}(p(D))$ is a component through $x^{\prime}$. Under the assumption $(*)$, one has: If $D$ is flat at $x$, then $D^{\prime}$ is flat at $x^{\prime}$.

Proof. Locally, flat sections are given by constant sections of $\left.H_{\mathbb{C}}^{1}\right|_{\Delta}=\mathbb{C}^{2} \times \Delta$, where $\Delta \subset T$ is a disk around a generic point of $T$. For a torsion section $D$, which corresponds to a section contained in $\mathbb{Q}^{2} \times \Delta$, assumption $(*)$ implies that $D^{\prime}$ is again torsion and hence flat. In order to prove the assertion, it is enough to prove that this holds true on an analytically dense set of flat local sections. However, since $\mathcal{C} \rightarrow T$ is not isotrivial, $\left.\left.R^{0} q_{*}\left(\Omega_{\mathcal{C} / T}\right)\right|_{\Delta} \subset H_{\mathbb{C}}^{1}\right|_{\Delta} \otimes \mathcal{O}$ does not admit flat sections and, therefore, $\Gamma\left(\Delta, R^{1} q_{*} \mathbb{C} / R^{1} q_{*} \mathbb{Z}\right)$ can be identified with the set of flat sections of $\left.\mathcal{C}\right|_{\Delta} \longrightarrow \Delta$. Under this identification, the subset of sections contained in $R^{1} q_{*} \mathbb{Q} / R^{1} q_{*} \mathbb{Z}$ is analytically dense in $\Gamma\left(\Delta, R^{1} q_{*} \mathbb{C} / R^{1} q_{*} \mathbb{Z}\right)$.

End of proof of Theorem A.1. Choose generic points $x, x^{\prime} \in \mathcal{C}$ with $p(x)=p\left(x^{\prime}\right)$ and assume that $x \in \mathcal{C}_{t}, t=q(x)$, is not torsion. Consider the two curves $E:=p\left(\mathcal{C}_{t}\right)$ and $E^{\prime}:=p\left(\mathcal{C}_{t^{\prime}}\right)$, $t^{\prime}=q(x)$, in $X$. Then we can assume that the two curves $E$ and $E^{\prime}$ intersect transversally in 


\section{Huybrechts}

$y:=p(x)=p\left(x^{\prime}\right) \in E \cap E^{\prime}$. Now choose a flat local analytic section $x \in D \subset \mathcal{C}$ of $q: \mathcal{C} \longrightarrow T$ such that $p(D)$ is tangent to $E^{\prime}$ at $y$. (This can always be achieved over points $t \in T$ with maximal VHS. Indeed a local calculation shows that every nonvertical tangent direction $v \in T_{x} \mathcal{C}$ in a point $x \in \mathcal{C}$ over $t$ can be integrated to a flat local section.) Then the component $D^{\prime} \subset p^{-1}(p(D))$ through $x^{\prime}$ is tangent to the fibre $\mathcal{C}_{t^{\prime}}, t^{\prime}=q\left(x^{\prime}\right)$. Since by Lemma A.4, $D^{\prime}$ is also flat, in fact $D^{\prime} \subset \mathcal{C}_{t^{\prime}}$.

But then $p^{-1}\left(E^{\prime}\right)$ contains $D$ and hence $D$ can be extended to an algebraic multi-section of $\mathcal{C} \rightarrow T$ through $x \in \mathcal{C}$ which, moreover, is flat locally around $x$ and nontorsion. Then, by Lemma A.3, $\mathcal{C} \rightarrow T$ is isotrivial, which is excluded by assumption on $\mathcal{C} \rightarrow T$.

\section{REFERENCES}

And05 Y. André, Motifs de dimension finie (d'après S.-I. Kimura, P. O'Sullivan ...), Séminaire Bourbaki. Vol. 2003/2004. Astérisque No. 299 (2005), Exp. No. 929, viii, 115-145.

AS08 M. Artebani and A. Sarti, Non symplectic automorphisms of order 3 on K3 surfaces, Math. Ann. 342 (2008), 903-921. http://dx.doi.org/10.1007/s00208-008-0260-1

AST11 M. Artebani, A.S̃arti and S. Taki, K3 surfaces with non-symplectic automorphisms of prime order, Math. Z. 268 (2011), 507-533; with an appendix by Shigeyuki Kondō. http://dx.doi. org/10.1007/s00209-010-0681-x

BV04 A. Beauville and C. Voisin, On the Chow ring of a K3 surface, J. Algebraic Geom. 13 (2004), 417-426. http://dx.doi.org/10.1090/S1056-3911-04-00341-8

Blo80 S. Bloch, Lectures on algebraic cycles, Duke Univ. Math. Series, IV (1980).

BKL76 S. Bloch, A. Kas and D. Lieberman, Zero cycles on surfaces with $p_{g}=0$, Compositio Math. 33 (1976), 135-145.

BS83 S. Bloch and V. Srinivas, Remarks on correspondences and algebraic cycles, Amer. J. Math. 105 (1983), 1235-1253.

BHT11 F. Bogomolov, B. Hassett and Y. Tschinkel, Constructing rational curves on K3 surfaces, Duke Math. J. 157 (2011), 535-550. http://dx.doi.org/10.1215/00127094-1272930

BT00 F. Bogomolov and Y. Tschinkel, Density of rational points on elliptic K3 surfaces, Asian J. Math. 4 (2000), 351-368.

BT05 F. Bogomolov and Y. Tschinkel, Rational curves and points on K3 surfaces, Amer. J. Math. 127 (2005), 825-835.

BP09 P. Brosnan and G. Pearlstein, Zero loci of admissible normal functions with torsion singularities, Duke Math. J. 150 (2009), 77-100. http://dx.doi.org/10.1215/00127094-2009-047

Cha12 F. Charles, The Tate conjecture for K3 surfaces over finite fields, Invent. Math., to appear, arXiv:1206.4002 (2012).

Cha13 F. Charles, Progrès récents sur les fonctions normales (d'après Green-Griffiths, BrosnanPearlstein, M. Saito, Schnell...), Séminaire Bourbaki (2013), arXiv:1301.7235 (2013).

Che02 X. Chen, A simple proof that rational curves on K3 are nodal, Math. Ann. 324 (2002), 71-104.

CL13 X. Chen and J. Lewis, Density of rational curves on K3 surfaces, Math. Ann. 356 (2013), 331-354. http://dx.doi.org/10.1007/s00208-012-0848-3

Dil09 J. Dillies, Order 6 non-symplectic automorphisms of K3 surfaces, arXiv:0912.5228 (2009).

Ful98 W. Fulton, Intersection theory, Volume 2 of Ergebnisse der Mathematik und ihrer Grenzgebiete 3, Folge, Springer-Verlag, Berlin, (1998).

GS10 A. Garbagnati and A. Sarti, On symplectic and non-symplectic automorphisms of K3 surfaces, arXiv:1006.1604 (2010). 


\section{Curves AND CyCles ON K3 SuRfaCes}

Gor12 S. Gorchinskiy, Generation of modules and transcendence degree of zero-cycles, arXiv:1210.0233 (2012).

Gre98 M. Green, Higher Abel-Jacobi maps, Proceedings of the International Congress of Mathematicians, Vol. II (Berlin, 1998), Doc. Math. Extra Vol. II (1998), 267-276.

GGP04 M. Green, P. Griffiths and K. Paranjape, Cycles over fields of transcendence degree 1, Michigan Math. J. 52 (2004), 181-187. http://dx.doi.org/10.1307/mmj/1080837742

GG05 M. Green and P. Griffiths, On the tangent space to the space of algebraic cycles on a smooth algebraic variety, Annals of Mathematics Studies, 157, Princeton University Press (2005).

GG03 M. Green and P. Griffiths, An interesting 0-cycle, Duke J. Math. 119 (2003), 261-313.

GP03 V. Guletskii and C. Pedrini, Finite dimensional motives and the conjecture of Beilinson and Murre, K-theory 30 (2003), 1-21. http://dx.doi.org/10.1023/B:KTHE. 0000019787.69435. 89

HT00 B. Hassett and Y. Tschinkel, Abelian fibrations and rational points on symmetric products, Internat. J. Math. 11 (2000), 1163-1176. http://dx.doi.org/10.1142/S0129167X00000544

Huy13 D. Huybrechts, Lectures on K3 surfaces, http://www.math.uni-bonn.de/people/huybrech/ K3.html

Jan07 U. Jannsen, On finite-dimensional motives and Murre's conjecture, in 'Algebraic cycles and Motives' Vol. 2, London Math. Soc. Lecture Note Ser. 344, Cambridge Univ. Press (2007), $112-142$.

Ka03 B. Kahn, Équivalences rationnelle et numérique sur certaines variétés de type abélien sur un corps fini, Ann. Sci. École Norm. Sup. (4) 36 (2003), 977-1002. http://dx .doi .org/10.1016/ j.ansens.2003.02.002

KMP07 B. Kahn, J. Murre and C. Pedrini, On the transcendental part of the motive of a surface, in 'Algebraic cycles and Motives' Vol. 2, London Math. Soc. Lecture Note Ser. 344, Cambridge Univ. Press (2007), 143-202. http://dx.doi.org/10.1017/CB09781107325968.009

Ker06 M. Kerr, A survey of transcendental methods in the study of Chow groups of 0-cycles, in 'Mirror Symmetry V' (Lewis, Yui and Yau, eds.), AMS/IP Stud. Adv. Math. 38 (2006), 295-350.

Ker08 M. Kerr, Higher Abel-Jacobi maps for 0-cycles, J. K-Theory 2 (2008), 41-101. http://dx. doi.org/10.1017/is007011012jkt003

Kim05 S. Kimura, Chow groups are finite dimensional, in some sense, Math. Ann. 331 (2005), 173201. http://dx.doi.org/10.1007/s00208-004-0577-3

KMPS10 A. Klemm, D. Maulik and R. Pandharipande, E. Scheidegger, Noether-Lefschetz theory and the Yau-Zaslow conjecture, J. Amer. Math. Soc. 23 (2010), 1013-1040. http://dx.doi.org/ 10.1090/S0894-0347-2010-00672-8

Lec86 F. Lecomte, Rigidité des groupes de Chow, Duke Math. J. 53 (1986), 405-426.

LL12 J. Li, C. Liedtke, Rational curves on K3 surfaces, Invent. Math. 188 (2012), 713-727. http: //dx.doi.org/10.1007/s00222-011-0359-y

Lie13 C. Liedtke, Supersingular K3 surfaces are unirational, arXiv:1304.5623 (2013).

Mac04 C. Maclean, Chow groups of surfaces with $h^{2,0} \leqslant 1$, C. R. Math. Acad. Sci. Paris 338 (2004), 55-58. http://dx.doi.org/10.1016/j.crma.2003.10.039

Mad13 K. Madapusi Pera, The Tate conjecture for K3 surfaces in odd characteristic, arXiv:1301.6326 (2013).

Mau12 D. Maulik, Supersingular K3 surfaces for large primes, arXiv:1203.2889 (2012).

Mil82 J. Milne, Zero cycles on algebraic varieties in nonzero characteristic: Rojtman's theorem, Compositio Math. 47 (1982), 271-287.

Mil07 J. Milne, The Tate conjecture over finite fields, arXiv:0709.3040 (2007).

MM83 S. Mori and S. Mukai, The uniruledness of the moduli space of curves of genus 11, Alg. Geometry (Tokyo/Kyoto, 1982), 334-353, Lecture Notes in Math., 1016, Springer, Berlin, (1983). 


\section{Huybrechts}

Mum68 D. Mumford, Rational equivalence of 0-cycles on surfaces, J. Math. Kyoto Univ. 9 (1968) 195-204.

OG10 K. O'Grady, Double covers of EPW-sextics, arXiv:1007.4952v3 (2010).

OG13 K. O'Grady, Moduli of sheaves and the Chow group of K3 surfaces, arXiv:1205.4119. J. Math. Pures Appl. (2013), to appear.

Ped12 C. Pedrini, On the finite dimensionality of a K3 surface, Manuscripta Math. 138 (2012), 59-72. http://dx.doi.org/10.1007/s00229-011-0483-4

Roj80 A. Rojtman, The torsion of the group of 0-cycles modulo rational equivalence, Ann. Math. (2) 111 (1980), 553-569.

Sai08 M. Saito, Hausdorff property of the Néron models of Green, Griffiths and Kerr, arXiv:0803.2771v4 (2008).

Sch87 C. Schoen, Zero cycles modulo rational equivalence for some varieties over fields of transcendence degree one, Algebraic geometry, Bowdoin, 1985 (Brunswick, Maine, 1985), Proc. Sympos. Pure Math. 46, Part 2, (1987), 463-473.

Tat94 J. Tate, Conjectures on algebraic cycles in $\ell$-adic cohomology, Motives (Seattle, WA, 1991), Proc. Sympos. Pure Math. 55, Part 1, AMS (1994), 71-83.

Tik80 A. Tikhomirov, Geometry of the Fano surface of a double $\mathbb{P}^{3}$ branched in a quartic, Izv. Akad. Nauk SSSR Ser. Mat. 44 (1980), 415-442.

Voi96 C. Voisin, Remarks on zero-cycles of self-products of varieties, Moduli of vector bundles (Sanda, 1994; Kyoto, 1994), Lecture Notes in Pure and Appl. Math. 179, Dekker, New York, (1996), 265-285.

Voi99 C. Voisin, Some results on Green's higher Abel-Jacobi map, Ann. of Math. 149 (1999), 451-473.

Voi02 C. Voisin, Théorie de Hodge et géométrie algébrique complexe, Cours spécialisés 10, SMF (2002).

Voi12a C. Voisin, Bloch's conjecture for Catanese and Barlow surfaces, arXiv:1210.3935 (2012).

Voi12b C. Voisin, Rational equivalence of 0-cycles on K3 surfaces and conjectures of Huybrechts and O'Grady, arXiv:1208.0916 (2012).

Voi12c C. Voisin, Symplectic involutions of K3 surfaces act trivially on $\mathrm{CH}_{0}$, Documenta Math. 17 (2012) 851-860.

Wel81 G. Welters, Abel-Jacobi isogenies for certain types of Fano threefolds, Mathematical Centre Tracts 141 (1981).

Yin13 Q. Yin, On a result of Green and Griffiths, arXiv:1302.6378 (2013).

D. Huybrechts huybrech@math.uni-bonn.de

Mathematisches Institut, Universität Bonn, Endenicher Allee 60, 53115 Bonn, Germany 